
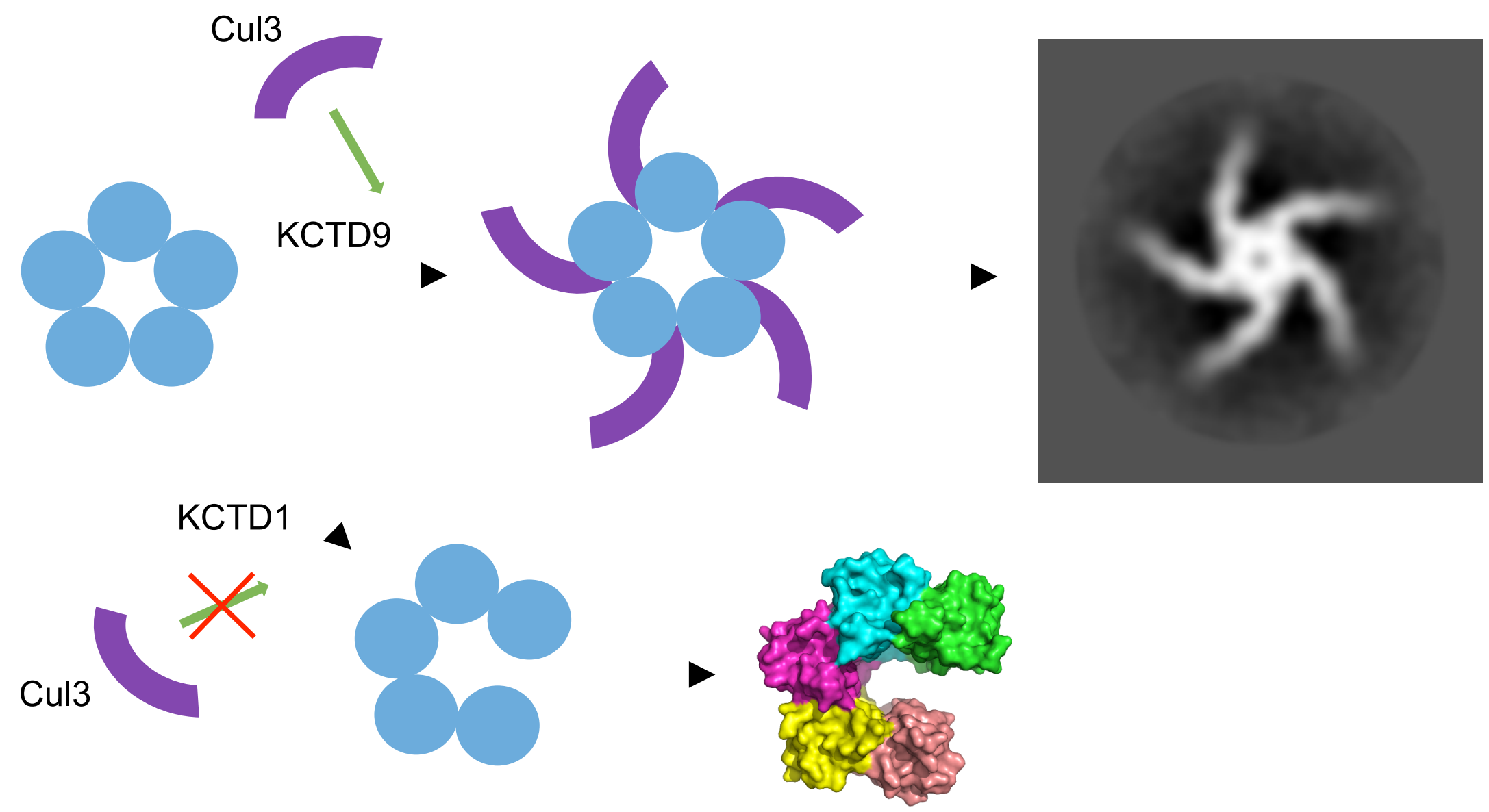


\section{Structural insights into KCTD protein assembly and Cullin3 recognition}

Alan X. Ji ${ }^{1}$, Anh Chu, Tine Kragh Nielsen, Samir Benlekbir, John L. Rubinstein and Gilbert G.

$$
\text { Privé }{ }^{1,2,3}
$$

${ }^{1}$ Department of Biochemistry, University of Toronto, 1 Kings College Circle, Toronto, Ontario M5S 1A8, Canada

${ }^{2}$ Department of Medical Biophysics, University of Toronto, 610 University Avenue, Toronto, Ontario M5G 2M9, Canada

${ }^{3}$ Princess Margaret Cancer Centre, Campbell Family Institute for Cancer Research, University Health Network, 101 College Street, Toronto, Ontario M5G 1L7, Canada

Correspondence should be addressed to G.G.P.

Gilbert G. Privé

Princess Margaret Cancer Centre

Toronto Medical Discovery Tower

101 College Street

Toronto, Ontario, Canada M5G 1L7

phone: (416) 581-7541

fax: (416) 581-7562

email: prive@uhnres.utoronto.ca 


\begin{abstract}
Cullin3 (Cul3)-based ubiquitin E3 ligase complexes catalyze the transfer of ubiquitin from an E2 enzyme to target substrate proteins. In these assemblies, the C-terminal region of Cul3 binds Rbx1/E2 ubiquitin, while the N-terminal region interacts with various BTB domain proteins that serve as substrate adaptors. Previous crystal structures of the homodimeric BTB proteins KLHL3, KLHL11 and SPOP in complex with the N-terminal domain of Cul3 revealed the features required for $\mathrm{Cul3}$ recognition in these proteins. A second class of BTB-domain containing proteins, the KCTD proteins, are also Cul3 substrate adaptors, but these do not share many of the previously identified determinants for $\mathrm{Cul3}$ binding. We report the pentameric crystal structures of the KCTD1 and KCTD9 BTB domains, and identify plasticity in the KCTD1 rings. We find that the KCTD proteins 5, 6, 9, and 17 bind to Cul3 with high affinity, while the KCTD proteins 1 and 16 do not have detectable binding. Finally, we confirm the 5:5 assembly of KCTD9/Cul3 complexes by electron cryomicroscopy and provide a molecular rationale for BTB-mediated Cul3 binding specificity in the KCTD family.
\end{abstract}




\section{Introduction}

The human genome encodes 183 BTB (Bric-à-brack, Tram-track, Broad complex) domain containing proteins, most of which cluster into families that share distinct domain architectures. This defines the ZBTB family (BTB-Zinc Finger proteins; 43 members), the KLHL family (BTB-BACK-Kelch proteins; 49 members), the $\mathrm{T} 1 / \mathrm{Kv}$ family $(\mathrm{T} 1-\mathrm{K}+$ channel integral membrane proteins; 27 members), and the KCTD family (Potassium Channel Tetramerization Domain proteins; 25 members) [1]. Most of the ZBTB and KLHL proteins contain a "long form" of the BTB domain that includes an N-terminal $\beta$-strand $/ \alpha$-helix extension to the core fold that mediates homodimerization, while the T1 and KCTD family members contain a "short form" of the domain made up exclusively of the core fold [1]. The full-length KCTD proteins consist of a single short form N-terminal BTB domain and variable C-terminal sequences with no evidence of transmembrane domains. In contrast to the tetramers seen in the $\mathrm{T} 1 / \mathrm{Kv}$ protein, the crystal structure of KCTD5 revealed a homopentamer [2], and while two members of the KCTD family, KCTD7 and KCNRG, have been shown to interact with $\mathrm{Kv}$ channels [3,4], this is not a general feature of the family. Thus, the KCTD proteins are functionally and structurally distinct from the $\mathrm{T} 1 / \mathrm{Kv}$ proteins $[2,5,6]$.

Many, but not all, BTB proteins are substrate adaptors in ubiquitin-based Cullin3-Ring Ligase (CRL3) complexes [7-11], and have a central role in assembling the functional complexes. Structures of N-terminal fragments of Cullin3 (Cul3) with KLHL3, KLHL11 and SPOP (a MATH-BTB-BACK protein) have revealed the basis for binding between BTB-BACK domains and Cul3 [12-14]. In these proteins, the Kelch domain (in the KLHL proteins) or MATH domain (in SPOP and SPOPL) bind to substrate proteins and target these for 
ubiquitination. These works and others identified two key signatures in BTB-BACK domains for Cul3 binding: a $\varphi$-X-E motif in the loop between BTB helix 3 and beta strand 4 (the variable "vloop"), and a 3-box motif in the BACK domain immediately following the BTB domain [12-15].

Many KCTD proteins, including KCTDs 5, 6, 7, 11, and BTBD10, interact with Cul3 [6,16-22] (Table S1) and target specific substrates for CRL3-based ubiquitination and subsequent proteosomal degradation $[5,6]$. However, these KCTD proteins do not contain a $\varphi$-XE motif, nor do they contain a 3-box/BACK or BACK-like domain, suggesting that a different set of determinants are used for Cul3 recognition in these proteins.

In this study, we present the structural, biophysical and biochemical characterization of the BTB domains of the KCTD family proteins 1, 5, 6, 9, 16, and 17, including crystal structures of $\mathrm{KCTD} 1^{\mathrm{BTB}}$ and $\mathrm{KCTD} 9^{\mathrm{BTB}}$. KCTD 1, 5, 6, 9 and $17 \mathrm{BTB}$ domains form homopentamers, and while some, such as KCTD9, appear to be associated into rigid rings, others such as KCTD1, may be more dynamic. We find that Cul3 binding is a common but not a universal property of the KCTD family [21], as KCTD proteins 5, 6, 9, and 17 associated with Cul3 with affinities ranging from 2-80 nM, while we could not detect any binding of Cul3 to KCTDs 1 and 16. We confirm that KCTD9 forms 5:5 complexes with Cul3, and based on our structural analysis, we propose determinants for $\mathrm{Cul} 3$ binding that are unique to this family of proteins.

\section{Results}

Structures of the KCTD1 ${ }^{\mathrm{BTB}}$ and $\mathrm{KCTD9} 9^{\mathrm{BTB}}$ pentamers 
We solved the crystal structures of the BTB domains of KCTD9 and KCTD1 (Fig. 1 and Table 1). KCTD $9^{\mathrm{BTB}}$ forms a near-perfect 5-fold symmetric pentamer, similar to the previously published KCTD5 structure [2]. Notably, the v-loop region between $\alpha 2$ and $\beta 3$ (equivalent to the $\alpha 3-\beta 4$ loop in long-form BTB domain) was disordered in all five chains of this structure, and we could not model residues 122-128 from this loop region into the KCTD9 electron density maps.

We obtained two different crystal forms of $\mathrm{KCTD}^{\mathrm{BTB}}{ }^{\mathrm{B}}$. The form 1 crystals diffracted to $2.3 \AA$ resolution and contained $10 \mathrm{KCTD} 1^{\mathrm{BTB}}$ chains arranged in two closed pentamers, which we refer to as the form $1 \mathrm{a}$ and $1 \mathrm{~b}$ pentamers. $\mathrm{KCTD}^{\mathrm{BTB}}$ form 2 crystals were resolved to $1.8 \AA$ resolution and contained $5 \mathrm{KCTD}^{\mathrm{BTB}}$ chains arranged in an open ring. At the single chain level, all of the $15 \mathrm{KCTD} 1 \mathrm{BTB}$ structures are highly similar with an average pairwise C $\alpha$ RMSD of $0.7 \AA$. The only variable region is the v-loop between $\alpha 2$ and $\beta 3$, which adopts different conformations in KCTD1 form 2 chains A and D but is otherwise similar in all of the other 13 chains (Fig. 1F). Larger differences are seen at the quaternary level, with nearly ideal 5-fold symmetry in $\mathrm{KCTD}^{\mathrm{BTB}}$ form $1 \mathrm{a}$, while the form $1 \mathrm{~b}$ pentamer displays a slight screw offset around a pseudo-5-fold axis. The form 2 assembly has an even more pronounced dislocations, and as a result, the fifth subunit is not in contact with the first subunit.

The differences in each pentamer were quantified by a superposition analysis of the subunits within each assembly (Fig. 2). For ease of discussion, the chains in each of the BTB pentamers discussed here are referred to as chains A through E. In the regular KCTD9 ${ }^{\mathrm{BTB}}$ pentamer, the rotation angles between adjacent subunits range from $70.9^{\circ}$ to $72.3^{\circ}$, with vertical translation values between -0.2 to $0.1 \AA$, supporting the assignment of near-ideal C5 symmetry in this assembly. The five interfaces between adjacent subunits areas highly similar, and all have surface areas within $698-743 \AA^{2}$. Similar values were obtained from an analysis of the 
$\mathrm{KCTD5}^{\mathrm{BTB}}$ pentamer (PDB ID 3DRZ) [2]. $\mathrm{KCTD1}^{\mathrm{BTB}}$ form 1a also has near-perfect $\mathrm{C} 5$ symmetry, with inter-subunit rotation angles ranging from $69^{\circ}$ to $76^{\circ}$ and vertical translation values of -0.1 to $0.14 \AA$. All five interchain buried surface areas are within 794 and $845 \AA^{2}$. In contrast, the $\mathrm{KCTD} 1^{\mathrm{BTB}}$ form $1 \mathrm{~b}$ pentamer adopts a less regular structure, as evidenced by a gap developing at the E-A subunit interface. $\mathrm{KCTD}^{\mathrm{BTB}}$ form $1 \mathrm{~b}$ has rotation angles of $66-72^{\circ}$ and an average translations of $-2.4 \AA$ for 4 of the 5 interfaces, but with a rotation of $82^{\circ}$ and a vertical translation at $9 \AA$ for the operation from chain $\mathrm{E}$ to chain $\mathrm{A}$. This results in a lower buried surface area of $493 \AA^{2}$ for the E-A interface, compared to $790-867 \AA^{2}$ for the other four interfaces in this pentamer. In the $\mathrm{KCTD} 1{ }^{\mathrm{BTB}}$ form 2 open pentamer, the rotation angles between adjacent monomers is much lower and ranges from $55^{\circ}$ to $67^{\circ}$ for the four interfaces that are in contact. As in the $1 \mathrm{~b}$ structure, there is an intersubunit translation of -1.7 to $-4.3 \AA$ for the subunits that are in contact. Thus, this assembly can be roughly described as having a pseudo 6fold symmetry with a screw component, but with one subunit missing. An inspection of the crystal lattice reveals that a putative $6^{\text {th }}$ site cannot be occupied due to crystal packing blocking the "empty" site. A $6^{\text {th }}$ subunit can be modeled into the form 2 assembly, but it is not possible to extend the helical chain with a $7^{\text {th }}$ subunit, since a $7^{\text {th }}$ subunit would clash with the first subunit (i.e. the $\sim 12 \AA$ pitch of the helix is substantially less than the $\sim 45 \AA$ height of the individual subunits). In both the $\mathrm{KCTD} 1^{\mathrm{BTB}}$ form $1 \mathrm{~b}$ and form 2 assemblies, the smaller intersubunit rotation angles and the offset in the translation along the rotation axis suggests that this is the more "relaxed" form of the interface between the subunits, and that the slightly larger rotation values and near-zero screw dislocations required for strict C5 symmetry and ring closure introduces a degree of strain in the form 1a pentamer. 
In order to further analyze the similarities and differences between the subunit interfaces in the KCTD BTB domain pentamers, we compared all of the in-contact neighbour pairs with a reference pair consisting of chains A and B from each pentamer (Fig. 2D). In this analysis, we superposed chain A from the reference dimer onto one of the chains of a target dimer, and calculated the C $\alpha$ RMSD between the reference chain B and the non-fit companion chain of the target pair. If the intersubunit interfaces are similar between the reference and target dimers, the RMSD values between reference chain B and the target subunit will be low. In the case of a structure with strict C5 symmetry the resulting RMSD value would be zero. For the three KCTD1 pentamers, we used chains A and B from form 1a as the reference dimer.

The RMSD values obtained in this way for the chains of the KCTD5 pentamer ranged from 2-3 $\AA$, while both KCTD9 and KCTD1 form 1a revealed more regular interchain interfaces with RMSD values of less than $1.2 \AA$. The four conserved KCTD1 form $1 \mathrm{~b}$ interfaces have similar values, however, as expected, the atypical interface between chains E-A generated a much larger RMSD value of $11 \AA$. The values for $\mathrm{KCTD} 1^{\mathrm{BTB}}$ form 2 are higher than other structures, ranging from 2.9-4.9 $\AA$ for the interfaces that are in physical contact, indicating that there are significant variations in the intersubunit contacts when the chains are not constrained to a C5-symmetric pentamer.

\section{Oligomeric state of KCTD proteins in solution}

The size of the KCTD proteins were analyzed in solution by analytical size exclusion chromatography (SEC) (Fig. 3). $\mathrm{KCTD}^{\mathrm{BTB}}$ elutes with a $\mathrm{MW}_{\text {app }}$ of $54 \mathrm{kDa}$, which formally corresponds to an assembly of 4.3 chains, while $\mathrm{KCTD}^{\mathrm{BTB}}$ elutes with a $\mathrm{MW}_{\text {app }}$ of $68 \mathrm{kDa}$, or 
5.5 chains per assembly. $\mathrm{KCTD}^{\mathrm{BTB}}$ elutes at a size corresponding to $57 \mathrm{kDa}$ ( 4.7 chains) and $\mathrm{KCTD} 17^{\mathrm{BTB}}$ at $79 \mathrm{kDa}$ (5.6 chains). Full length KCTD5 elutes at approximately $164 \mathrm{kDa}$, which corresponds to 6.2 chains. Given the accuracy of the method and the effect of shape on the elution volumes, these results do not conclusively prove pentameric assemblies, but they are consistent with the pentamers seen in the crystal structures of KCTD1, 5 and 9.

The oligomeric state of KCTD BTB domains was also studied by glutaraldehyde crosslinking followed by SDS-PAGE (Fig. 4). The BTB domains of KCTD1 and KCTD6 gave clear crosslinking patterns resulting in bands corresponding to monomers, dimers, trimers, tetramers, and pentamers. KCTD $9^{\mathrm{BTB}}$ samples behaved similarly, although larger species were observed above the pentamer band. Cross-linking results with other KCTD BTB domains were not conclusive, presumably due to an unfavourable distribution of lysine residues on the protein surfaces.

\section{Interactions with $\mathrm{Cul3}$}

We used size-exclusion chromatography (SEC) to characterize the binding of Cul3 to the KCTDs (Fig. 3). Cul3 $3^{1-381}$ elutes with a $\mathrm{MW}_{\text {app }}$ of $51 \mathrm{kDa}$, which is close to the expected mass of the $43 \mathrm{kDa}$ monomer. Mixtures of the BTB domains from KCTD5, 6, 9, and 17 with Cul3 $3^{1-381}$ resulted in shifts towards larger sizes, indicating that these proteins are able to form complexes with Cul3 in solution. Smaller shifts were seen when point mutations F128A in KCTD5 ${ }^{\text {BTB }}$ and $\mathrm{V} 125 \mathrm{~A}$ in $\mathrm{KCTD} 9^{\mathrm{BTB}}$ were introduced, indicating that these mutations partly disrupted the Cul3 interactions. These mutations were selected based on the expected Cul3 contact sites [22] and our modelling; see below. Neither of these mutations appears to disrupt the fold of the proteins 
since the individual peaks eluted similarly to the wild-type proteins. Notably, no shifts were observed when $\mathrm{Cul} 3^{1-381}$ was mixed with $\mathrm{KCTD} 1^{\mathrm{BTB}}$.

The binding of KCTD proteins to Cul3 was quantified by isothermal titration calorimetry (ITC) (Fig. 5). In all cases where complex formation was observed, the binding stoichiometry corresponded to one $\mathrm{Cul} 3$ chain to each $\mathrm{KCTD}$ chain. $\mathrm{KCTD}^{\mathrm{BTB}}$ bound $\mathrm{Cul}^{1-381}$ with a $\mathrm{Kd}$ of $43 \mathrm{nM}$ while full length KCTD5 bound five times more strongly with a $\mathrm{Kd}$ of $8.5 \mathrm{nM}$. The dissociation constants for $\mathrm{KCTD}^{\mathrm{BTB}}$ and $\mathrm{KCTD} 9^{\mathrm{BTB}}$ were $2.2 \mathrm{nM}$ and $11 \mathrm{nM}$, respectively. The titration with KCTD6 had a low enthalpy change and a Kd of $2 \mathrm{nM}$, consistent with a recently published value of $1.1 \mathrm{nM}$ for KCTD6 to Cul3 binding [21]. In line with our SEC experiments, $\mathrm{KCTD}^{\mathrm{BTB}}$ F128A bound nearly one thousand fold more weakly to Cul3 than the wild-type protein, and the $\mathrm{KCTD} 9^{\mathrm{BTB}}$ V125A substitution reduced binding by 32 -fold. Again, as seen in the SEC experiment, no binding was observed when the BTB domains of KCTD1 or 16 were titrated into solutions of $\mathrm{Cul}^{1-381}$.

\section{Structure of the KCTD9/Cul3 complex}

We generated models of a $\mathrm{KCTD} 9^{\mathrm{BTB}} / \mathrm{Cul}^{31-381}$ complex based on the crystal structure of the 2:2 KLHL3/Cul3 complex [14]. The KLHL3 BTB domain was aligned onto the KCTD9 BTB domain structure, and the resulting position of the Cul3 N-terminal domain from the experimental structure did not produce any significant clashes within the KCTD pentamer. This was repeated five times to produce a 5:5 $\mathrm{KCTD} / \mathrm{Cul} 3$ complex. Both proteins were extended to their full-length structures by including the C-terminal domain from KCTD5 (PDB 3DRX), the full length Cul1/Rbx1 complex (PDB 1LDK), and the E2 Ub structure (PDB 1FBV) as 
previously described for the dimeric $\mathrm{BTB} / \mathrm{Cul} 3$ complexes $[13,14]$. The resulting complex is a symmetric 5-fingered claw with the Cul3 chains forming curved extensions extending from the central KCTD pentamer (Fig. 6A), in agreement with a previous study [22]. The C-terminal domains of the KCTD protein, which presumably bind to the substrates targeted for $\mathrm{Ub}$ modification, extend outwardly from the "palm" position of the claw, and are located on the same face as the Rbx1/E2 Ub moieties. Similar suprafacial arrangements of Ub and substrate binding sites are consistently observed in 2:2 E3 ligase complexes [13,14,23].

In order to test this model experimentally, complexes of $\mathrm{KCTD} 9^{\mathrm{BTB}} / \mathrm{Cul} 3^{1-381}$ were prepared and analyzed by single particle electron cryomicroscopy (cryo-EM). Particles corresponding to 5-pointed pinwheels were readily observed. Averaged, but not symmetrized, images were consistent with the model, and the application of 5-fold symmetry further improved the quality of the images (Fig. 6B-F). A volumetric rendition of the model was aligned onto the cryo-EM images with excellent agreement (Fig. 6G,H). The projected view along the 5-fold axis reveals a core assembly containing the KCTD9 BTB domain pentamer, with five curving, extended arms corresponding to the Cul3 chains.

\section{Model of the KCTD/Cul3 interface}

The KCTD BTB domain structures described here, combined with solution binding data, cryo-EM results and previously determined $\mathrm{BTB} / \mathrm{Cul3}$ crystal structures, provide a strong foundation for generating an accurate atomic model for the $\mathrm{KCTD} / \mathrm{Cul} 3$ complexes (Fig. 6, 7).

Cul3 makes contacts with two adjacent BTB subunits, and we describe these distinct and non-overlapping interfaces as the primary and distal contact surfaces (Fig. 7). The KCTD 
primary contact surface is equivalent to the primary contact surface seen in other BTB/Cullin complexes, including the SPOP/KLHL family, Skp1 and Elongin C (Fig. 8, 9). In this interface, the primary BTB contact surface is made up of the $\alpha 2 / \mathrm{v}$-loop $/ \beta 3$ region and the $\mathrm{C}$-terminal half of helix $\alpha 4$ and the following loop. These elements engage the $\mathrm{H} 2$ helix and the $\mathrm{N}$ terminus of the H5 helix of Cul3 (Fig. 7). Our mutational data support an important contribution from a conserved aromatic residue in helix $\alpha 4$ for Cul3 binding [22]: all KCTD proteins with the exception of KCTD19 have either a Phe or Tyr residue at the position equivalent to F128 in KCTD5 (Fig. 7, S1), and the F128A mutation in KCTD5 reduces the Cul3 affinity (Fig. 3, 5). However, the non-Cul3 binders, including KCTD1, 12, 15 and 16, also have an aromatic residue at this position.

In contrast with the primary contact surface, the distal contact surface is not conserved amongst the BTB families. In the SPOP/KLHL, Skp1 and Elongin C families, the distal contact surface involves non-BTB regions of the proteins, and these interact with the C-terminal end of Cul3 helices $\mathrm{H} 2$ and $\mathrm{H} 5$. This distal surface consists of the BACK domain residues in the SPOP/KLHL family, F-box residues in Skp1, and C-terminal/SOCS-box residues in ElonginC. In the KCTD proteins, however, the distal contact surface is unique in that it involves residues from the adjacent BTB subunit in the pentameric ring (Fig. 7, 9) [22]. The BTB elements of this distal interface include helix $\alpha 1$ and the loop following $\alpha 3$ (Fig. 7C). Furthermore, the KCTD distal contact surface interacts with a different surface of Cul3, consisting of the N-terminus of the $\mathrm{H} 1$ helix and one face of the $\mathrm{H} 2$ helix (Fig. 9B). In particular, Cul3 helix $\mathrm{H} 2$ is wedged between adjacent KCTD BTB subunits in the ring, and contributes a stripe of aromatic residues F54, Y58 and Y62 to the primary interface, and a stripe of polar residues E56, R59, N60, T63 
and H67 to the distal interface, while residues E55 and L66 contact both the primary and distal BTB surfaces.

We highlight two additional features of the KCTD primary contact surface that are likely to be important for Cul3 binding. First, the electrostatic potential of putative $\mathrm{Cul} 3$ binding sites of the known KCTD and modeled KCTD structures indicates a clear difference between Cul3 binders and non-binders. Cul3 contains a positively charged patch on its BTB-binding surface, and accordingly, complimentary acidic patches are seen on the primary contact surfaces of KCTDs 5, 6, 9, and 17 (Fig. 7, 8). In contrast, KCTD1 and 16 have positively charged surfaces in the equivalent areas. KCTD1 has an Arg residue at the Cul3 Y58 contact position, resulting in a large electropositive potential in the equivalent Cul3 contact region of KCTD1 (Fig. 8A). Other KCTD proteins have large hydrophobic residues at this position. The negatively charged Cul3 binding pocket observed on KCTD5, 6, 9, and 17 is also found in dimeric BTB proteins that bind Cul3, such as KLHL3, KLHL11, and SPOP (Fig. 8).

Second, the v-loop in the KCTD BTB proteins is an important determinant in Cul3 binding (Fig. 9A). In the dimeric long-form BTB proteins, this loop contains the conserved $\varphi$-XE motif required for Cul3 binding. A comparison of the KLHL11 and SPOP structures in the absence and presence of $\mathrm{Cul} 3$ shows that $\mathrm{Cul} 3$ binding induces a disorder-order transition in this loop $[12,13]$, and similar restructuring of this loop is expected in other Cul3 binders. The $\varphi-\mathrm{X}-\mathrm{E}$ sequence motif is not present in KCTD proteins, but the structure of this loop remains important. Here, the V125A KCTD9 mutation in the v-loop weakens Cul3 binding (Fig. 3, 5). The KCTD $9^{\mathrm{BTB}}$ crystallographic maps have very weak electron density for all five chains in this region, indicating structural disorder in this loop. Consequently, residues 122-128 from loop are not modeled in our KCTD9 structures. Similarly, residues from this loop were also not modeled 
in some of the KCTD5 crystal structures [2], and a molecular dynamics study of KCTD11 also revealed flexibility in this loop [21]. We suggest that the V125A KCTD9 mutation alters the local structure and/or the dynamics of the v-loop so that the mutant can no longer bind Cul3 efficiently. We expect that this loop adopts a fixed conformation in a Cul3 complex, and that residue V125 of KCTD9 makes contact with a hydrophobic surface of Cul3. Overall, there is low sequence conservation in this loop in the KCTD proteins and no simple pattern differentiates the Cul3 binders from the non-binders (Fig. 7, S1). Instead, based on the structures of KCTD1 relative to the Cul3 binders KCTD5, SPOP and KLHL3, we observe that this loop is less dynamic and positioned differently versus the others (Fig. 1F, Fig. S2). We propose that KCTD1 does not bind Cul3 in part because the KCTD1 surface is fairly rigid and is not complementary to the Cul3 surface. As we do not have an experimental structure for the KCTD16 BTB domain, we cannot predict if a similar mechanism is involved with this non-binder.

Overall, we suggest that a KCTD family member protein will bind Cul3 if it contains an appropriate negatively charged patch in the binding pocket and has a dynamic v-loop which can sample the "lower" conformational state, as seen in other BTB/Cul3 crystal structures.

\section{Relationships between KCTD proteins}

We constructed a phylogeny based the KCTD BTB domains sequences (Fig. 10, Fig. S1), and in general, the results are comparable with previously reported trees for the family $[5,6]$. We included additional human BTB sequences from proteins with structures available in the PDB, including T1 domains from voltage-gated potassium channels and BTB domains from KLHL3 and KLHL11, SPOP and two members of the BTB-ZF family (BCL6 and PLZF). These serve to 
root the cluster of KCTD sequences, and provide information about the relationships between the different BTB families. The inclusion of voltage-gated potassium channels clearly showed that the KCTD family is distinct from the T1 group [6], however Clade G (BTBD10 and KCTD20) is more closely related to the dimeric long-form BTB domains from the KLHL and ZBTB families. The binding of Cul3 correlates well with the assigned clades, with members of Clades A and F emerging as non-Cul3 binders. Although not strongly supported by bootstrap values, it appears likely that Cul3 binding was an early event in the expansion of the BTB family, and that this ability was lost in the T1 domains and in the KCTD Clades A and F. Contrary to the argument presented by Smaldone et al. [21], the bootstrap values from our work or from references [5, 6] are not sufficient to argue whether Clades A and F independently lost Cul3 binding, or whether this occurred in a common ancestor to these groups.

\section{Discussion}

The three different conformations for the KCTD1 BTB pentamers in our crystal structures reveal a spectrum of states ranging from a symmetric pentamer in KCTD1 form 1a, to an asymmetrical closed pentamer in form $1 \mathrm{~b}$, to an open $\mathrm{C}$-shaped ring in form 2 . The biological significance of these closed and open states of the KCTD1 ring remains to be explored, however, given that $\mathrm{Cul} 3$ binds to adjacent chains in the pentameric ring, any dynamism in the KCTD1

ring may an additional factor that disfavours Cul3 binding in this protein. This mechanism may be at work in other KCTDs that do not bind Cul3. A more uniform C5 symmetry occurs in the Cul3 binders KTCD5 and KTCD9. Thus, ring dynamics may be an additional factor that 
influences Cul3 binding in addition to the surface electrostatics and the characteristics of helix $\alpha 4$ and the $\alpha 2 / \beta 3$ v-loop.

It is not known whether other KCTD proteins can also transition between open and closed states in solution, but the variability in the KCTD1 BTB pentamers suggests that not all members of the KCTD family adopt strictly symmetrical pentameric rings. Our SEC results may reflect some variability in other members of the KCTD family, and the BTB domains from KCTD6, 11 and 12 have been characterized as a tetramers by SEC-MALS [21].

Other regions of KCTD proteins may also influence the characteristics and dynamics of the KCTD rings. In the case of KCTD5, the C-terminal domain forms an independent homopentamer which is distinct from the N-terminal BTB domain [2]. A protein with two domains that can independently self-assemble would stabilize the pentameric state, and presumably produces a regular binding surface that favours Cul3 binding. This hypothesis is supported by our ITC data where full length KCTD5 binds Cul3 fivefold stronger than the BTB domain alone.

The assignment of KCTD proteins into Cul3-binders and non-binders provides important mechanistic insight into the biology of these proteins. The KCTD11 tumor suppressor (also known as REN) is a biologically well-characterized KCTD/Cul3 system. KCTD11 normally promotes the CRL3-based ubiquitination and subsequent degradation of HDAC1. This in turn downregulates Hedgehog and Gli signaling. Mutations or deletions causing loss of KCTD11 function is associated with medulloblastoma [24-26], and recent studies have shown that the other Clade B proteins KCTD6 and KCTD21, also function in HDAC1 regulation [16]. 
We demonstrate Cul3 binding for KCTD9, confirming an earlier prediction [6]. KCTD9 is involved in liver injury during hepatic viral infections, and is upregulated in hepatic natural killer cells upon viral infection proportional to the severity of infection. This results in upregulated CD69 expression, cytotoxicity, interferon- $\gamma$ secretion, and downregulation of the NKG2A receptor [27-29]. A recent study has also shown KCTD9 may interact with influenza A proteins [30]. The natural biological role of KCTD9 in the absence of viral infection, and the function of KCTD9/Cul3 binding in viral liver injury, remains unknown. We expect that at least some of these functions involve $\mathrm{CRL} 3^{\mathrm{KCTD} 9}$-based substrate ubiquitination.

The biology of the Cul3-binding KCTD proteins are mostly understood in terms of their substrate binding specificity in CRL3 ubiquitin ligase complexes. Remarkably, several of the non-Cul3 binders are also involved ubiquitin processes, but these involve different types of E3 complexes. For example, KCTD1, a protein structurally characterized in this study, has been shown to interact with the armadillo repeats of $\beta$-catenin via its BTB domain to mediate the suppression of Wnt signaling [31]. KCTD1 enhances the ubiquitination and degradation of $\beta$ catenin, however in this case, the E3 ligase is based on $\beta$-Transducin repeat Containing Protein $(\beta-\operatorname{TrCP})$ and not $\mathrm{Cul3} \mathrm{[32].}$

Other KCTD proteins are not currently connected with ubiquitin biology. KCTD8, 12 and 16 (Clade F) are a subgroup of closely related proteins that function as auxiliary $\mathrm{GABA}_{\mathrm{B}}$ receptor subunits by directly binding to the receptor and an associated $G$ protein $[33,34]$. Clade $F$ KCTD proteins have recently been shown to modulate $\mathrm{GABA}_{\mathrm{B}}$ receptor response by affecting Gprotein activation [35]. KCTD12 inhibits G $\beta \gamma$ binding to Kir3 channels, causing a desensitization of the channel to $\mathrm{GABA}_{\mathrm{B}}$ receptor signaling, which is important for suppressing proliferation in gastrointestinal stromal tumors [34,36]. In contrast, KCTD 8 and 16 work to 
inhibit the desensitization caused by KCTD12 [37]. KCNRG is one of two KCTD proteins known to interact with Kv channels, and functions by sequestering Kv channels in the cytoplasm, possibly through competitive binding of KCNRG BTB domains to $\mathrm{Kv}$ T1 domains during channel assembly $[4,38]$.

Ultimately, the elucidation of the biological roles for the members of the KCTD family depends on gaining a molecular understanding of these proteins. Clearly, deciphering the stoichiometry and assembly properties of the KCTD proteins is an important part of understanding their roles as adaptors. The characterization of pentameric rings in KCTD1 and KCDT9, as well as the geometry of the Cul3 complexes, expand our understanding of these proteins. 


\section{Materials and Methods}

\section{Cloning, protein expression and purification}

Human KCTD cDNAs were obtained from the Hospital for Sick Children SPARC centre (Toronto) and from the human ORFeome library via the laboratory of Dr. Igor Stagljar [39]. The NCBI accession numbers are: KCTD1 [NM_001136205], KCTD5 [NM_018992], KCTD6 [NM_153331], KCTD9 [NM_017634], KCTD16 [NM_020768] and KCTD17 [NM_001282684]. PCR fragments were cloned into E. coli expression vectors. KCTD $1^{\text {BTB }}$ (residues 29-103), $\mathrm{KCTD}^{\mathrm{BTB}}$ (residues 43-145), KCTD9 ${ }^{\mathrm{BTB}}$ (residues 89-191), $\mathrm{KCTD}^{\mathrm{BTB}}$ (residues 22-130), and $\mathrm{KCTD} 17^{\mathrm{BTB}}$ (residues 24-141) were cloned into the pMCSG7 vector by ligation independent cloning [40]. The pMCSG7 vector contains an N-terminal 6xHis tag followed by a TEV cleavage site. USER cloning [41] was employed to clone $\mathrm{KCTD}^{\mathrm{BTB}}$ (residues 10-115) into the pET-2ST vector, which contains an N-terminal SUMO fusion protein and 6xHis tag followed by a TEV cleavage site. $\mathrm{KCTD}^{\mathrm{FL}}$ (residues 1-234) was cloned into the PSJ5 vector by restriction enzyme cloning. $\mathrm{KCTD}^{\mathrm{FL}}$ DNA was amplified with a 5' BamHI overhang and 3' NotI overhangs. The PSJ5 vector contains an N-terminal thioredoxin protein and a 6xHis tag followed by a TEV cleavage site. Cul3 residues $1-381\left(\mathrm{Cul}^{\mathrm{NTD}}\right)$ was cloned into the previously described pMCSG7 vector.

Proteins were expressed in BL21(DE3) Codon Plus E. coli grown in TB media at $37{ }^{\circ} \mathrm{C}$ until $\mathrm{OD}_{600 \mathrm{~nm}}$ of 0.6 , the cultures were then cooled to $15{ }^{\circ} \mathrm{C}$ and induced with $0.5 \mathrm{mM}$ IPTG and harvested after $12 \mathrm{~h}$. The proteins were purified by NiNTA chromatography, followed by TEV digestion to remove the purification tag and size exclusion chromatography, as previously reported. [14]. 


\section{Crystallization, data collection and processing}

Crystallization trials were performed using purified $\mathrm{KCTD}^{\mathrm{BTB}}$ proteins in 96 well sitting drop plates using an Art-Robbins Phoenix drop setter. Equivalent volumes of purified protein solutions at 5-30 mg/mL in $20 \mathrm{mM}$ Tris $\mathrm{pH} 7.5,150 \mathrm{mM} \mathrm{NaCl}, 1 \mathrm{mM}$ TCEP were mixed with Qiagen JCSG Core I-IV crystallization screen solutions and equilibrated with the screen solutions. Crystal lead conditions were optimized in 24 well hanging drop plates.

$\mathrm{KCTD} 1^{\mathrm{BTB}}$ was crystallized in two crystal forms, however, both forms were sometimes obtained under the identical conditions. Form 1 crystals of $\mathrm{KCTD} 1^{\mathrm{BTB}}$ optimized for data collection were grown using $10 \mathrm{mg} / \mathrm{mL} \mathrm{KCTD1}^{\mathrm{BTB}}, 0.1 \mathrm{M} \mathrm{MES} \mathrm{pH}$ 6, 5\% w/v PEG 1000, 30\% w/v PEG 600, and 10\% v/v glycerol. The crystals were cryo-protected using 5\% v/v ethylene glycol prior to flash-freezing. $\mathrm{KCTD}^{\mathrm{BTB}}$ form 2 crystals were grown using $25 \mathrm{mg} / \mathrm{mL}$ $\mathrm{KCTD}^{\mathrm{BTB}}$, 0.1 M MES pH 5, 10\% PEG 6000, and cryo-protected using 10\% ethylene glycol. KCTD $9^{\mathrm{BTB}}$ crystals were grown at $4{ }^{\circ} \mathrm{C}$ using $25 \mathrm{mg} / \mathrm{mL}$ KCTD9 ${ }^{\mathrm{BTB}}, 0.17 \mathrm{M}$ lithium citrate, and 16\% w/v PEG 3350. KCTD9 ${ }^{\mathrm{BTB}}$ crystals were cross-linked with a 5\% glutaraldehyde solution via vapor diffusion [42] before cryo-protection with 10\% ethylene glycol and flash-freezing.

Native data were collected at the Advanced Photon Source (APS) beamline 17-ID. In addition, an iodine derivative for the KCTD1 form 2 crystal was obtained by the vaporizing iodine labeling method [43] and anomalous data were collected on a Bruker MicroStar diffractometer with $\mathrm{Cu} \mathrm{K} \alpha$ radiation. The iodine derivative dataset was solved using Phenix [44] Auto-Sol MR-SAD with a KCTD5 BTB monomer search model from PDB ID 3DRZ [2] followed by manual rebuilding. A partially built four-chain model was then used for the refinement of the native KCTD1 form 2 dataset, and a fifth chain was identified and built into the electron density. A monomer from the KCTD1 form 2 structure was used as an MR search 
model for the KCTD1 form 1 dataset, which had partial pseudo-merohedral twinning and was refined with a twin fraction of 0.2 . The KCTD9 crystal structure was solved using Phenix molecular replacement with a search model based on a KCTD5 BTB domain monomer. All structures were refined using Phenix Refine [44].

\section{Modeling and structure analyses}

A locally modified version of the program SUPPOS (B.W. Dijkstra) was used to calculate the superposition parameters between the KCTD subunits. Interface areas were calculated using PISA [45]. Backbone atom RMSD values and figures were generated with PyMol [46].

Homology models of the BTB domains of KCTD6, 16, and 17 were generated using ITasser [47-49]. The KCTD/Cul3 complexes were generated by superposing the BTB domains of KCTD proteins onto the BTB domain of KLHL3 from the KLHL3/Cul3 structure (PDB ID 4HXI) [14]. The full KCTD5 based CRL3 ubiquitinylation complex was generated by docking the $\mathrm{Cul}^{\mathrm{NTD}}$ from the initial KCTD5/Cul3 model onto the full length structure of Cul1 from the Skp1/Cul1 complex. KCTD/Cul3 interface contacts were estimated using the CoCo-Maps web server [50]. KCTD and Cul3 surface electrostatics were generated using PyMol. Structure based sequence alignments were performed using the PDBeFold web server [51]. KCTD BTB domain sequences were aligned with MUSCLE [52] and phylogenies were constructed with PHYML [53]. 


\section{Solution characterization}

Analytical size exclusion chromatography (SEC) experiments were performed using purified proteins on a $25 \mathrm{~mL}$ Tricorn S200a column. Glutaraldehyde crosslinking experiments were performed using purified KCTD BTB domains in $20 \mathrm{mM}$ HEPES pH 7.5, $150 \mathrm{mM} \mathrm{NaCl}$ at $0.5-1.5 \mu \mathrm{g} / \mu \mathrm{L}$ with $0.1-0.2 \% \mathrm{v} / \mathrm{v}$ glutaraldehyde in $10 \mu \mathrm{L}$ sample volumes. Reactions were quenched at specified time points with $10 \mu \mathrm{L}$ of Tris/SDS sample buffer, boiled, and loaded onto 12\% Tricine gels. Isothermal titration calorimetry (ITC) experiments were performed using purified KCTD and Cul3 proteins at $25{ }^{\circ} \mathrm{C}$ using a VP-ITC Micro Calorimeter. Cul3 ${ }^{1-381}$ was loaded into the reservoir at 5-20 $\mu \mathrm{M}, \mathrm{KCTD}$ protein at 10 fold higher concentrations was then injected into the reservoir. Data were processed using Origin, and binding isotherms were calculated based on a one-site binding model.

\section{Electron cryomicroscopy}

Holey carbon film coated EM grids with regular arrays of 500-800 nm holes were prepared by

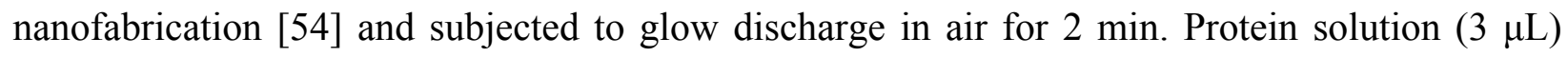
was applied to grids with a Vitrobot grid preparation robot (FEI) and allowed to equilibrate for 5 $\mathrm{s}$ then blotted from both sides for $20 \mathrm{~s}$ and frozen in liquid cryogen $(50 \%$ ethane and $50 \%$ propane). Grids were transferred to a Gatan 626 specimen holder and imaged with an FEI F20 electron microscope equipped with a field emission gun and a Gatan K2 Summit camera and operating a $200 \mathrm{kV}$. The DDD was used in counting movie mode with $5 \mathrm{e}$-/pixel/sec for $15 \mathrm{sec}$ and $0.5 \mathrm{sec} /$ frame. This exposure rate resulted in $1.2 \mathrm{e}^{-} / \AA^{2} /$ frame on the specimen. Seven movies were acquired with defocuses between 2 and $4 \mathrm{~m}$ and movie frames were aligned using 
alignframes_lmbfgs [55] and averaged. Averaged frames were used to calculate contrast transfer function (CTF) parameters with CTFFIND3 [56] and to select particle images with Relion [57]. 256 particle images were selected and classified into $102 \mathrm{D}$ classes. C5 symmetry was applied to two of the classes with the program apply3dsymmetry (J. Rubinstein, unpublished software)

\section{Accession numbers}

The structure factors and atomic coordinates for $\mathrm{KCTD}^{\mathrm{BTB}}$ form 1 and form 2, and $\mathrm{KCTD} 9^{\mathrm{BTB}}$ were deposited into the Protein Data Bank with PDB IDs 5BXB, 5BXD and 5BXH, respectively.

\section{Acknowledgements}

We thank Alan Wong for preliminary work on this project. This work was supported by grants to GGP from the CCSRI, CRS and CIHR. JLR was supported by CIHR MOP 81294 and the Canada Research Chairs program. This research was funded in part by the Ontario Ministry of Health and Long Term Care (OMOHLTC). The funders had no role in study design, data collection and analysis, decision to publish, or preparation of the manuscript. Use of the IMCACAT beamline 17-ID at the Advanced Photon Source was supported by the companies of the Industrial Macromolecular Crystallography Association through a contract with HauptmanWoodward Medical Research Institute. Use of the Advanced Photon Source was supported by the U.S. Department of Energy, Office of Science, Office of Basic Energy Sciences, under Contract No. DE-AC02-06CH11357. 


\section{Keywords:}

KCTD; BTB domain; Cullin3; E3 Ubiquitin Ligase Complex; Protein-Protein interactions

\section{Abbreviations used:}

BTB Bric-à-brack, Tram-track, Broad complex

KCTD $\quad \underline{K}^{+}$Channel Tetramerization Domain

Cryo-EM Electron cryomicroscopy

SEC size-exclusion chromatography

Cul3 Cullin3

ITC isothermal titration calorimetry 


\section{References}

[1] Stogios PJ, Downs GS, Jauhal JJS, Nandra SK, Privé GG. Sequence and structural analysis of BTB domain proteins. Genome Biol 2005;6:R82. doi:10.1186/gb-2005-6-10r82.

[2] Dementieva IS, Tereshko V, McCrossan Z a., Solomaha E, Araki D, Xu C, et al. Pentameric Assembly of Potassium Channel Tetramerization Domain-Containing Protein 5. J Mol Biol 2009;387:175-91. doi:10.1016/j.jmb.2009.01.030.

[3] Krabichler B, Rostasy K, Baumann M, Karall D, Scholl-Bürgi S, Schwarzer C, et al. Novel Mutation in Potassium Channel related Gene KCTD7 and Progressive Myoclonic Epilepsy. Ann Hum Genet 2012;76:326-31. doi:10.1111/j.1469-1809.2012.00710.x.

[4] Ivanov D V., Tyazhelova T V., Lemonnier L, Kononenko N, Pestova a. a., Nikitin E a., et al. A new human gene KCNRG encoding potassium channel regulating protein is a cancer suppressor gene candidate located in 13q14.3. FEBS Lett 2003;539:156-60. doi:10.1016/S0014-5793(03)00211-4.

[5] Liu Z, Xiang Y, Sun G. The KCTD family of proteins: structure, function, disease relevance. Cell Biosci 2013;3:45. doi:10.1186/2045-3701-3-45.

[6] Skoblov M, Marakhonov A, Marakasova E, Guskova A, Chandhoke V, Birerdinc A, et al. Protein partners of KCTD proteins provide insights about their functional roles in cell differentiation and vertebrate development. BioEssays 2013;35:586-96. doi:10.1002/bies.201300002.

[7] Bennett EJ, Rush J, Gygi SP, Harper JW. Dynamics of cullin-RING ubiquitin ligase network revealed by systematic quantitative proteomics. Cell 2010;143:951-65. doi:10.1016/j.cell.2010.11.017.

[8] Furukawa M, He YJ, Borchers C, Xiong Y. Targeting of protein ubiquitination by BTBCullin 3-Roc1 ubiquitin ligases. Nat Cell Biol 2003;5:1001-7. doi:10.1038/ncb1056.

[9] Geyer R, Wee S, Anderson S, Yates J, Wolf D a. BTB/POZ domain proteins are putative substrate adaptors for cullin 3 ubiquitin ligases. Mol Cell 2003;12:783-90. doi:10.1016/S1097-2765(03)00341-1.

[10] Pintard L, Willis JH, Willems A, Johnson J-LF, Srayko M, Kurz T, et al. The BTB protein MEL-26 is a substrate-specific adaptor of the CUL-3 ubiquitin-ligase. Nature 2003;425:311-6. doi:10.1038/nature01959. 
[11] Xu L, Wei Y, Reboul J, Vaglio P, Shin T-H, Vidal M, et al. BTB proteins are substratespecific adaptors in an SCF-like modular ubiquitin ligase containing CUL-3. Nature 2003;425:316-21. doi:10.1038/nature01985.

[12] Canning P, Cooper CDO, Krojer T, Murray JW, Pike ACW, Chaikuad A, et al. Structural basis for Cul3 protein assembly with the BTB-Kelch family of E3 ubiquitin ligases. J Biol Chem 2013;288:7803-14. doi:10.1074/jbc.M112.437996.

[13] Errington WJ, Khan MQ, Bueler S a., Rubinstein JL, Chakrabartty A, Privé GG. Adaptor protein self-assembly drives the control of a cullin-RING ubiquitin ligase. Structure 2012;20:1141-53. doi:10.1016/j.str.2012.04.009.

[14] Ji AX, Privé GG. Crystal Structure of KLHL3 in Complex with Cullin3. PLoS One 2013;8:e60445. doi:10.1371/journal.pone.0060445.

[15] Zhuang M, Calabrese MF, Liu J, Waddell MB, Nourse A, Hammel M, et al. Structures of SPOP-Substrate Complexes: Insights into Molecular Architectures of BTB-Cul3 Ubiquitin Ligases. Mol Cell 2009;36:39-50. doi:10.1016/j.molcel.2009.09.022.

[16] De Smaele E, Di Marcotullio L, Moretti M, Pelloni M, Occhione MA, Infante P, et al. Identification and characterization of KCASH2 and KCASH3, 2 novel Cullin3 adaptors suppressing histone deacetylase and Hedgehog activity in medulloblastoma. Neoplasia 2011;13:374-85. doi:10.1593/neo.101630.

[17] Bayón Y, Trinidad AG, De La Puerta ML, Del Carmen Rodríguez M, Bogetz J, Rojas A, et al. KCTD5, a putative substrate adaptor for cullin3 ubiquitin ligases. FEBS J 2008;275:3900-10. doi:10.1111/j.1742-4658.2008.06537.x.

[18] Azizieh R, Orduz D, Van Bogaert P, Bouschet T, Rodriguez W, Schiffmann SN, et al. Progressive myoclonic epilepsy-associated gene KCTD7 is a regulator of potassium conductance in neurons. Mol Neurobiol 2011;44:111-21. doi:10.1007/s12035-011-8194-0.

[19] Lange S, Perera S, Teh P, Chen J. Obscurin and KCTD6 regulate cullin-dependent small ankyrin-1 (sAnk1.5) protein turnover. Mol Biol Cell 2012;23:2490-504. doi:10.1091/mbc.E12-01-0052.

[20] Kim W, Bennett EJ, Huttlin EL, Guo A, Li J, Possemato A, et al. Systematic and quantitative assessment of the ubiquitin-modified proteome. Mol Cell 2011;44:325-40. doi:10.1016/j.molcel.2011.08.025.

[21] Smaldone G, Pirone L, Balasco N, Di Gaetano S, Pedone EM, Vitagliano L. Cullin 3 Recognition Is Not a Universal Property among KCTD Proteins. PLoS One 2015;10:e0126808. doi:10.1371/journal.pone.0126808.

[22] Balasco N, Pirone L, Smaldone G, Di Gaetano S, Esposito L, Pedone EM, et al. Molecular recognition of Cullin3 by KCTDs: Insights from experimental and computational 
investigations. Biochim Biophys Acta - Proteins Proteomics 2014;1844:1289-98. doi:10.1016/j.bbapap.2014.04.006.

[23] Tang X, Orlicky S, Lin Z, Willems A, Neculai D, Ceccarelli D, et al. Suprafacial Orientation of the SCFCdc4 Dimer Accommodates Multiple Geometries for Substrate Ubiquitination. Cell 2007;129:1165-76. doi:10.1016/j.cell.2007.04.042.

[24] Correale S, Pirone L, Di Marcotullio L, De Smaele E, Greco A, Mazz D, et al. Molecular organization of the cullin E3 ligase adaptor KCTD11. Biochimie 2011;93:715-24. doi:10.1016/j.biochi.2010.12.014.

[25] Di Marcotullio L, Ferretti E, De Smaele E, Argenti B, Mincione C, Zazzeroni F, et al. $\mathrm{REN}(\mathrm{KCTD} 11)$ is a suppressor of Hedgehog signaling and is deleted in human medulloblastoma. Proc Natl Acad Sci U S A 2004;101:10833-8. doi:10.1073/pnas.0400690101.

[26] Canettieri G, Di Marcotullio L, Greco A, Coni S, Antonucci L, Infante P, et al. Histone deacetylase and Cullin3-REN(KCTD11) ubiquitin ligase interplay regulates Hedgehog signalling through Gli acetylation. Nat Cell Biol 2010;12:132-42. doi:10.1038/ncb2013.

[27] Chen T, Zhu L, Zhou Y, Pi B, Liu X, Deng G, et al. KCTD9 contributes to liver injury through NK cell activation during hepatitis B virus-induced acute-on-chronic liver failure. Clin Immunol 2013;146:207-16. doi:10.1016/j.clim.2012.12.013.

[28] Zhou Y, Pi B, Liu X, Zhang R, Deng G, Chen T, et al. Increased expression of KCTD9, a novel potassium channel related gene, correlates with disease severity in patients with viral hepatitis B. Zhonghua Gan Zang Bing Za Zhi 2008;16:835-9.

[29] Huang J, Xiao F, Yu H, Huang T, Huang H, Ning Q. Differential gene expression profiles in acute hepatic failure model in mice infected with MHV-3 virus intervened by antihepatic failure compound. J Huazhong Univ Sci Technol - Med Sci 2007;27:538-42. doi:10.1007/s11596-007-0516-0.

[30] Generous A, Thorson M, Barcus J, Jacher J, Busch M, Sleister H. Identification of putative interactions between swine and human influenza A virus nucleoprotein and human host proteins. Virol J 2014;11:2509. doi:10.1186/s12985-014-0228-6.

[31] Li X, Chen C, Wang F, Huang W, Liang Z, Xiao Y, et al. KCTD1 suppresses canonical Wnt signaling pathway by enhancing $\beta$-catenin degradation. PLoS One 2014;9:e94343. doi:10.1371/journal.pone.0094343.

[32] Aberle H, Bauer A, Stappert J, Kispert A, Kemler R. B-Catenin Is a Target for the Ubiquitin-Proteasome Pathway. EMBO J 1997;16:3797-804.

doi:10.1093/emboj/16.13.3797. 
[33] Schwenk J, Metz M, Zolles G, Turecek R, Fritzius T, Bildl W, et al. Native GABA(B) receptors are heteromultimers with a family of auxiliary subunits. Nature 2010;465:231-5. doi:10.1038/nature08964.

[34] Turecek R, Schwenk J, Fritzius T, Ivankova K, Zolles G, Adelfinger L, et al. Auxiliary GABAB receptor subunits uncouple $G$ protein $\beta \gamma$ subunits from effector channels to induce desensitization. Neuron 2014;82:1032-44. doi:10.1016/j.neuron.2014.04.015.

[35] Rajalu M, Fritzius T, Adelfinger L, Jacquier V, Besseyrias V, Gassmann M, et al. Pharmacological characterization of GABAB receptor subtypes assembled with auxiliary KCTD subunits. Neuropharmacology 2015;88:145-54. doi:10.1016/j.neuropharm.2014.08.020.

[36] Hasegawa T, Asanuma H, Ogino J, Hirohashi Y, Shinomura Y, Iwaki H, et al. Use of potassium channel tetramerization domain-containing 12 as a biomarker for diagnosis and prognosis of gastrointestinal stromal tumor. Hum Pathol 2013;44:1271-7. doi:10.1016/j.humpath.2012.10.013.

[37] Seddik R, Jungblut SP, Silander OK, Rajalu M, Fritzius T, Besseyrias V, et al. Opposite effects of KCTD subunit domains on GABA(B) receptor-mediated desensitization. J Biol Chem 2012;287:39869-77. doi:10.1074/jbc.M112.412767.

[38] Usman H, Mathew MK. Potassium channel regulator KCNRG regulates surface expression of Shaker-type potassium channels. Biochem Biophys Res Commun 2010;391:1301-5. doi:10.1016/j.bbrc.2009.11.143.

[39] Yang X, Boehm JS, Yang X, Salehi-Ashtiani K, Hao T, Shen Y, et al. A public genomescale lentiviral expression library of human ORFs. Nat Methods 2011;8:659-61. doi:10.1038/nmeth.1638.

[40] Stols L, Gu M, Dieckman L, Raffen R, Collart FR, Donnelly MI. A new vector for highthroughput, ligation-independent cloning encoding a tobacco etch virus protease cleavage site. Protein Expr Purif 2002;25:8-15. doi:10.1006/prep.2001.1603.

[41] Salomonsen B, Mortensen UH, Halkier B a. USER-derived cloning methods and their primer design. Methods Mol Biol 2014;1116:59-72. doi:10.1007/978-1-62703-764-8_5.

[42] Lusty CJ. A gentle vapor-diffusion technique for cross-linking of protein crystals for cryocrystallography. J Appl Crystallogr 1999;32:106-12.

doi:10.1107/S002188989801053X.

[43] Miyatake H, Hasegawa T, Yamano A. New methods to prepare iodinated derivatives by vaporizing iodine labelling (VIL) and hydrogen peroxide VIL (HYPER-VIL). Acta Crystallogr Sect D Biol Crystallogr 2006;62:280-9. doi:10.1107/S0907444905041909. 
[44] Adams PD, Afonine P V., Bunkóczi G, Chen VB, Davis IW, Echols N, et al. PHENIX: A comprehensive Python-based system for macromolecular structure solution. Acta Crystallogr Sect D Biol Crystallogr 2010;66:213-21. doi:10.1107/S0907444909052925.

[45] Krissinel E, Henrick K. Inference of Macromolecular Assemblies from Crystalline State. J Mol Biol 2007;372:774-97. doi:10.1016/j.jmb.2007.05.022.

[46] Schrödinger, LLC. The $\{$ PyMOL $\}$ Molecular Graphics System, Version 1.5.0.3 2013.

[47] Roy A, Kucukural A, Zhang Y. I-TASSER: a unified platform for automated protein structure and function prediction. Nat Protoc 2010;5:725-38. doi:10.1038/nprot.2010.5.

[48] Yang J, Yan R, Roy A, Xu D, Poisson J, Zhang Y. The I-TASSER Suite: protein structure and function prediction. Nat Methods 2014;12:7-8. doi:10.1038/nmeth.3213.

[49] Zhang Y. I-TASSER server for protein 3D structure prediction. BMC Bioinformatics 2008;9:40. doi:10.1186/1471-2105-9-40.

[50] Vangone A, Spinelli R, Scarano V, Cavallo L, Oliva R. COCOMAPS: A web application to analyze and visualize contacts at the interface of biomolecular complexes.

Bioinformatics 2011;27:2915-6. doi:10.1093/bioinformatics/btr484.

[51] Krissinel E, Henrick K. Secondary-structure matching (SSM), a new tool for fast protein structure alignment in three dimensions. Acta Crystallogr Sect D Biol Crystallogr 2004;60:2256-68. doi:10.1107/S0907444904026460.

[52] Edgar RC. MUSCLE: Multiple sequence alignment with high accuracy and high throughput. Nucleic Acids Res 2004;32:1792-7. doi:10.1093/nar/gkh340.

[53] Guindon S, Gascuel O. A simple, fast, and accurate algorithm to estimate large phylogenies by maximum likelihood. Syst Biol 2003;52:696-704. doi:10.1080/10635150390235520.

[54] Marr CR, Benlekbir S, Rubinstein JL. Fabrication of carbon films with 500nm holes for cryo-EM with a direct detector device. J Struct Biol 2014;185:42-7. doi:10.1016/j.jsb.2013.11.002.

[55] Rubinstein JL; Brubaker MA. Alignment of cryo-EM movies of individual particles by optimization of image translations. J Struct Biol 2015; In press.

[56] Mindell J a., Grigorieff N. Accurate determination of local defocus and specimen tilt in electron microscopy. J Struct Biol 2003;142:334-47. doi:10.1016/S1047-8477(03)000698 .

[57] Scheres SHW. RELION: Implementation of a Bayesian approach to cryo-EM structure determination. J Struct Biol 2012;180:519-30. doi:10.1016/j.jsb.2012.09.006. 


\section{Figure Legends}

Fig. 1. Structural comparison of the BTB domain assemblies of (A) KCTD5 (PDB ID 3DRZ) [2], (B) KCTD9, and the three different examples of the KCTD1 pentamers: (C) form 1a, (D) form $1 \mathrm{~b}$, and (E) form 2. Rotation axes that superpose one BTB chain onto the next are shown as solid black lines. A horizontal dashed black line indicates the N-terminal baseline for each KCTD assembly. (F) Superposition of all 15 KCTD1 BTB domains colored by secondary structure (helices red, sheets yellow, loops green). The outlier v-loops of form 2-Chain A and form 2Chain D are highlighted in violet and cyan, respectively.

Fig. 2. Quantification of KCTD BTB domain symmetry differences. (A) Rotation angles for superposing neighboring BTB chains. (B) Vertical translation along the rotation axes during neighboring chain superposition. (C) Interface area between neighboring BTB chains. (D) Symmetry comparison of KCTD structures. A reference dimer consisting of chains A-B from each structure is generated. Chain A is superposed sequentially to the remaining chains, and the no-fit RMSD between the reference Chain B and the corresponding chain is plotted.

Fig. 3. Analytical size exclusion chromatography of KCTD proteins. (A) The BTB domains of KCTD1, 6, 17 and the N-terminal Cul3 fragment. (B) KCTD1, 6, and 17 BTB domains were mixed with Cul3 and analyzed by SEC. (C) Wild-type KCTD9 BTB domain protein, and mutant V125A KCTD9 BTB domain were run alone and with Cul3. (D) Wildtype full length KCTD5, and mutant F128A full length KCTD5 were run alone and with Cul3. 
Fig. 4. Glutaraldehyde crosslinking of purified KCTD1, 6, and 9 BTB domains. Reactions were quenched at the indicated time points and resolved by SDS-PAGE. A schematic interpretation of the observed bands is shown on the right. Some species larger than pentamers are observed in the KCTD9 experiment.

Fig. 5. Isothermal titration calorimetry experiments using purified KCTD proteins titrated into $\mathrm{Cul3}^{1-381}$ solutions: (A) KCTD $1^{\mathrm{BTB}}$, (B) $\mathrm{KCTD}^{\mathrm{BTB}}$, (C) full length KCTD5, (D) mutant F128A full length KCTD5, (E) KCTD6 ${ }^{\mathrm{BTB}}$, (F) KCTD9 ${ }^{\mathrm{BTB}}$, (G) mutant V125A KCTD9 ${ }^{\mathrm{BTB}}$, (H) $\mathrm{KCTD}_{16}^{\mathrm{BTB}}$, (I) $\mathrm{KCTD} 17^{\mathrm{BTB}}$.

Fig. 6. Models of KCTD/Cul3 mediated E3 ligase complexes based on single particle cryo-EM images. (A) Full length KCTD5 modelled with full length Cul3, Rbx1, and E2 Ubiquitin. All five Cul3 chains curve up towards the C-terminal domain face of the KCTD pentamer, and position the E2-Ubiquitin chains in the proximity of the C-terminal domain of KCTD5. (B) An electron micrograph of a field of $\mathrm{KCTD} 9^{\mathrm{BTB}} / \mathrm{Cul} 3^{1-381}$ complexes, with selected particles circled in red. Scale bar: $500 \AA$. (C, D) Class average images. (E, F) Class average images after the application of 5-fold rotational symmetry, scale bar: $100 \AA$. (G) Surface rendition of the KCTD $9^{\mathrm{BTB}} / \mathrm{Cul} 3^{1-381}$ model, with the KCTD9 pentamer in black. (H) Overlay of outline of the model from panel G (cyan) with the experimentally determined class average from panel $\mathrm{E}$. 
Fig. 7. Analysis of the KCTD/Cul3 interface. (A) Structural differences between KCTD1, 5, and 9 that may affect Cul3 binding. Cul3 (translucent grey) binds to two adjacent chains in the BTB pentamers. The primary Cul3 interface is with KCTD Chain A (green) and the distal interface is with KCTD Chain E (pale green). Key residues are highlighted in KCTD1 (green), KCTD5 (orange), and KCTD9 (magenta) (see text). Three differences between the non-Cul3 binding KCTD1 and Cul3 binding KCTD5 and 9 are highlighted. First, KCTD1 contains Y114 when KCTD5 and 9 have Phe residues. Second, KCTD1 contains an R55 in place of F69 in KCTD5 and M115 in KCTD9. Third, the v-loop in KCTD1 is positioned higher than the equivalent loop in KCTD5. This loop is not resolved in the KCTD9 structure. The higher loop position in KCTD1 may clash with helix 2 and 4 in Cul3. (B) Predicted Cul3 residues involved in KCTD binding. On the left, $\mathrm{Cul} 3$ is shown as an electrostatic surface with residues highlighted in grey stick format. On the right, Cul3 is shown as a translucent grey ribbon. KCTD9 is shown as a white surface with electrostatic coloring in the binding site. All labeled residues belong to Cul3. The weakly positive surface charge of Cul3 is complimentary to the mostly negative surface charge on KCTD9. (C) Structure-based multiple sequence alignment of selected KCTD BTB domains. KCTD residues on the primary interface (Chain A) are shaded pink while distal interface residues (Chain E) are shaded grey. Predicted Cul3 contact residues are indicated with triangles. Arrows indicate the $\mathrm{KCTD} 5^{\mathrm{F} 128 \mathrm{~A}}$ and $\mathrm{KCTD} 9^{\mathrm{V} 125 \mathrm{~A}}$ mutations that weaken $\mathrm{Cul} 3$ binding. The v-loop is represented as a dashed line in the secondary structure schematic.

Fig. 8. Surface electrostatics of cullin binding proteins. The $\mathrm{N}$ terminal cullin helices are shown as a translucent grey cartoons in approximately the same orientation in all panels. The binding partner is represented as a white surface and colored by surface electrostatics at the cullin 
interface. (A) Surface electrostatics of KCTD1 (non-binder), 5, and 9 BTB domains at the expected Cul3 interface. (B) Predicted surface electrostatics of KCTD6, 16 (non-binder), and 17 BTB domains based on modeled structures. (C) Surface electrostatics of complexes from the dimeric BTB proteins KLHL3, KLHL11, and SPOP bound to Cul3 from co-crystal structures 4HXI, 4AP2 and 4EOZ, respectively. Keap1 surface electrostatics were generated from the Keap1 BTB structure and docking Cul3. (D) Surface electrostatics of Skp1-2 bound to Cul1 (PDB ID 1LDK), and Socs2-Elongin BC bound to Cul5 (PDB ID 4JHG).

Fig. 9. Sequence and structure based analysis of KCTD/Cul3 interaction. (A) Structure based sequence alignment of BTB domains from the KCTD family (KCTD5, and 9; short-form BTB) and MATH-BTB and KLHL families (SPOP, KLHL3, KLHL11, and Keap1; long-form BTB). The KCTD BTB residues predicted to form the primary interface with Cul3 are shaded pink and distal interface residues are shaded grey. The MATH-BTB and KLHL primary interface residues that contact $\mathrm{Cul} 3$ are shaded blue. In these proteins, the distal interface residues are in the 3box/BACK domain region that follows the BTB domain (not shown). The extended v-loop in KCTD family proteins is highlighted as a dashed line in the secondary structure schematic and a box in the sequence alignment.The conserved $\varphi$-X-E Cul3 binding motif in the v-loop of the MATH-BTB and KLHL families is highlighted by a box and is not present in the KCTD proteins. Secondary structure elements found only in the long-form BTB domains are colored dark blue. (B) Overlay of the KCTD9/Cul3 and KLHL3/Cul3 complexes. The Cul3 N-terminal domain is shown in grey surface. The primary and distal subunits of the KCTD5 BTB pentamer are shown in dark magenta. The KLHL3 BTB and BACK domains are in green (single chain). The BTB 
primary interfaces overlap, however the distal interfaces of the KCTD and KLHL proteins contact different surfaces of Cul3.

Fig. 10. Phylogenetic trees based on BTB domain sequences. Proteins known to bind to Cul3 are highlighted in green, and Cul3 non-binders are highlighted in red (Table S1). KCTD clades A-G are assigned according to Skoblov et al. [6]. 
Table 1. Data collection and refinement statistics.

\begin{tabular}{|c|c|c|c|}
\hline & KCTD1 form1 & KCTD1 form2 & KCTD9 \\
\hline Wavelength $(\AA)$ & 1.000 & 1.000 & 1.000 \\
\hline Resolution range ( $\AA$ ) & $\begin{array}{l}94.76-2.17 \\
(2.25-2.17)\end{array}$ & $\begin{array}{l}41.08-1.79 \\
(1.86-1.79)\end{array}$ & $\begin{array}{l}30.57-2.76 \\
(2.86-2.76)\end{array}$ \\
\hline Space group & $\mathrm{P} 2_{1}$ & $\mathrm{P} 2{ }_{1} 2_{1} 2_{1}$ & $\mathrm{P} 3{ }_{1} 21$ \\
\hline Unit cell $\left(\AA,{ }^{\circ}\right)$ & $\begin{array}{l}66.8983 .0694 .76 \\
9090.190\end{array}$ & $\begin{array}{l}46.0690 .84127 .69 \\
909090\end{array}$ & $\begin{array}{l}105.40105 .4097 .32 \\
9090120\end{array}$ \\
\hline Total reflections & $103198(10500)$ & $101188(9973)$ & $160526(15780)$ \\
\hline Unique reflections & $54500(5451)$ & $50819(5001)$ & $16426(1599)$ \\
\hline Multiplicity & $1.9(1.9)$ & $2.0(2.0)$ & $9.8(9.8)$ \\
\hline Completeness & $0.99(1.00)$ & $1.00(1.00)$ & $1.00(1.00)$ \\
\hline Mean I/sigma(I) & $12.37(2.23)$ & $16.99(2.38)$ & $10.09(2.46)$ \\
\hline Wilson B-factor $\left(\AA^{2}\right)$ & 35.7 & 27.93 & 93.48 \\
\hline R-merge & $0.03731(0.3951)$ & $0.02157(0.3003)$ & $0.05735(0.829)$ \\
\hline R-meas & $0.05276(0.5587)$ & $0.0305(0.4247)$ & $0.06064(0.875)$ \\
\hline CC1/2 & $0.999(0.82)$ & $1(0.865)$ & $0.999(0.926)$ \\
\hline $\mathrm{CC}^{*}$ & $1(0.949)$ & $1(0.963)$ & $1(0.981)$ \\
\hline $\begin{array}{l}\text { Reflections used in } \\
\text { refinement }\end{array}$ & $54500(5451)$ & $50806(5001)$ & $16372(1599)$ \\
\hline Reflections used for $R$-free & $1986(206)$ & $2479(229)$ & $817(82)$ \\
\hline R-work & $0.1995(0.3543)$ & $0.1863(0.2868)$ & $0.2462(0.4988)$ \\
\hline R-free & $0.2361(0.3606)$ & $0.2211(0.3217)$ & $0.2881(0.5328)$ \\
\hline CC(work) & $0.934(0.802)$ & $0.950(0.664)$ & $0.952(0.711)$ \\
\hline $\mathrm{CC}($ free $)$ & $0.897(0.745)$ & $0.940(0.552)$ & $0.908(0.635)$ \\
\hline $\begin{array}{l}\text { Number of non-hydrogen } \\
\text { atoms }\end{array}$ & 9133 & 4699 & 4025 \\
\hline macromolecules & 8950 & 4376 & 4017 \\
\hline waters & 268 & 328 & 0 \\
\hline Protein residues & 1070 & 524 & 506 \\
\hline RMS(bonds) $(\AA)$ & 0.004 & 0.019 & 0.005 \\
\hline RMS(angles) $\left(^{\circ}\right)$ & 0.85 & 1.62 & 1.09 \\
\hline $\begin{array}{l}\text { Ramachandran favored } \\
(\%)\end{array}$ & 93 & 93 & 94 \\
\hline $\begin{array}{l}\text { Ramachandran allowed } \\
(\%)\end{array}$ & 5.3 & 5.3 & 5.6 \\
\hline $\begin{array}{l}\text { Ramachandran outliers } \\
(\%)\end{array}$ & 1.4 & 1.4 & 0.21 \\
\hline Rotamer outliers (\%) & 3.7 & 2.1 & 6.1 \\
\hline Clashscore & 8.39 & 8.52 & 6.57 \\
\hline Average B-factor $\left(\AA^{2}\right)$ & 58.22 & 49.96 & 118.73 \\
\hline macromolecules & 58.32 & 50.4 & 118.83 \\
\hline solvent & 53.33 & 44.09 & 71.35 \\
\hline Number of TLS groups & 1 & 5 & 5 \\
\hline
\end{tabular}


A

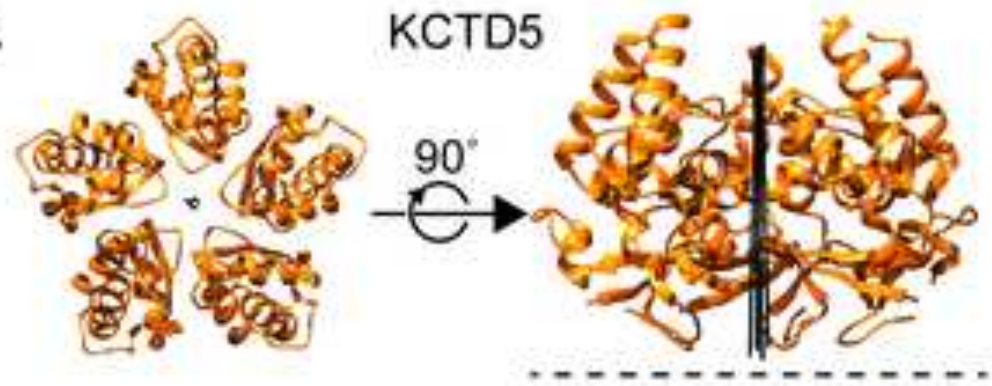

C

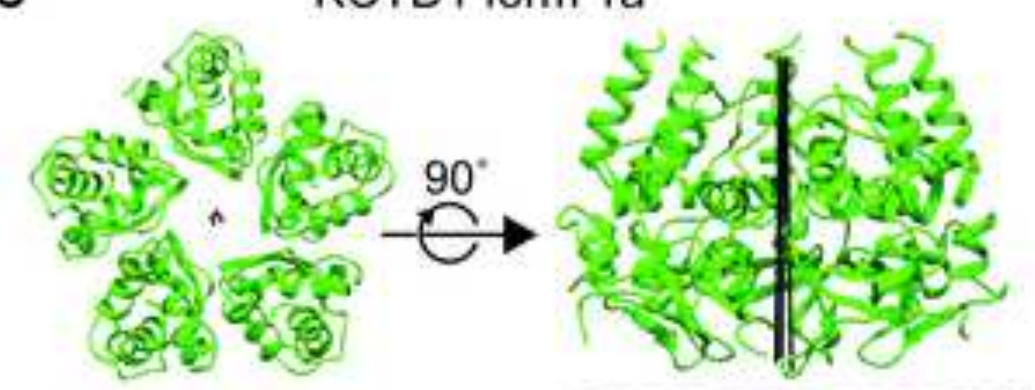

E

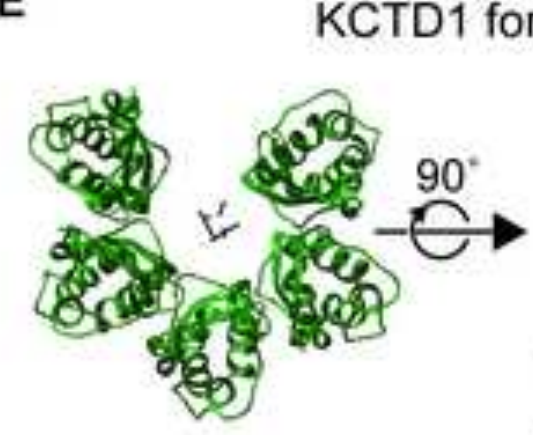

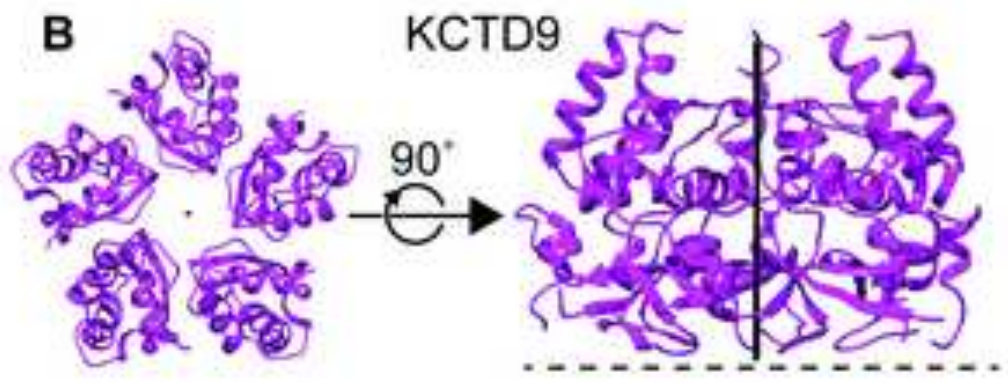

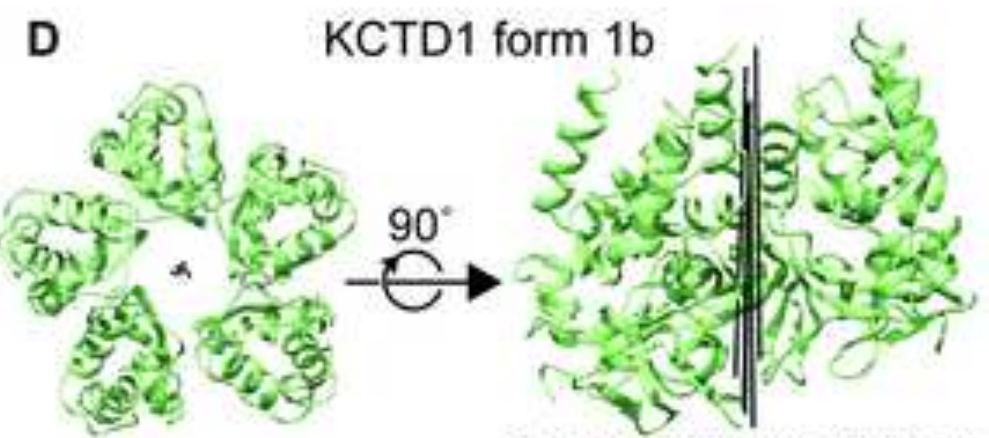

F

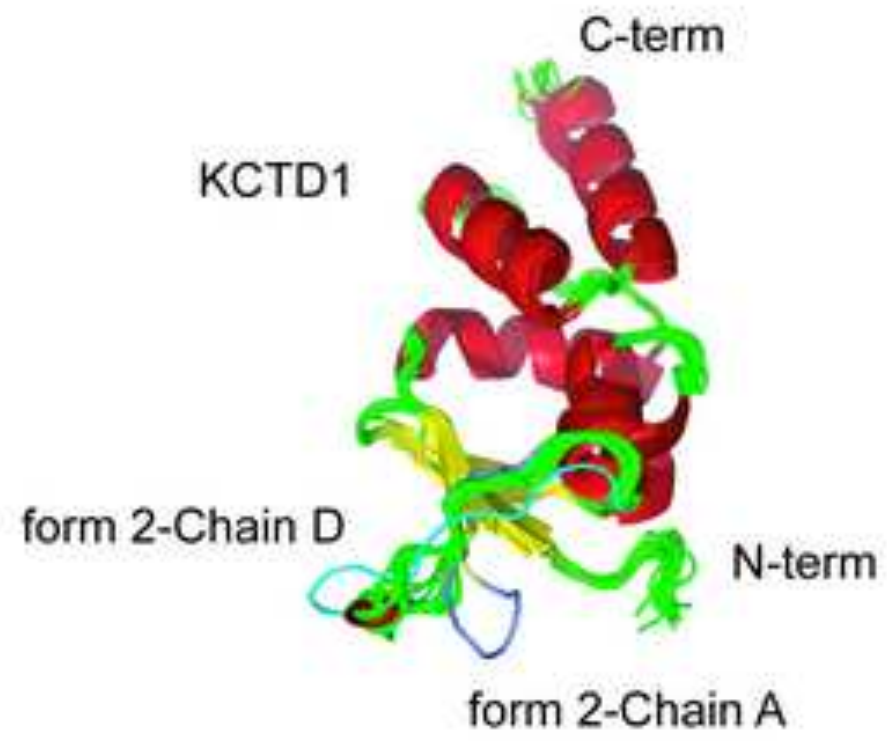




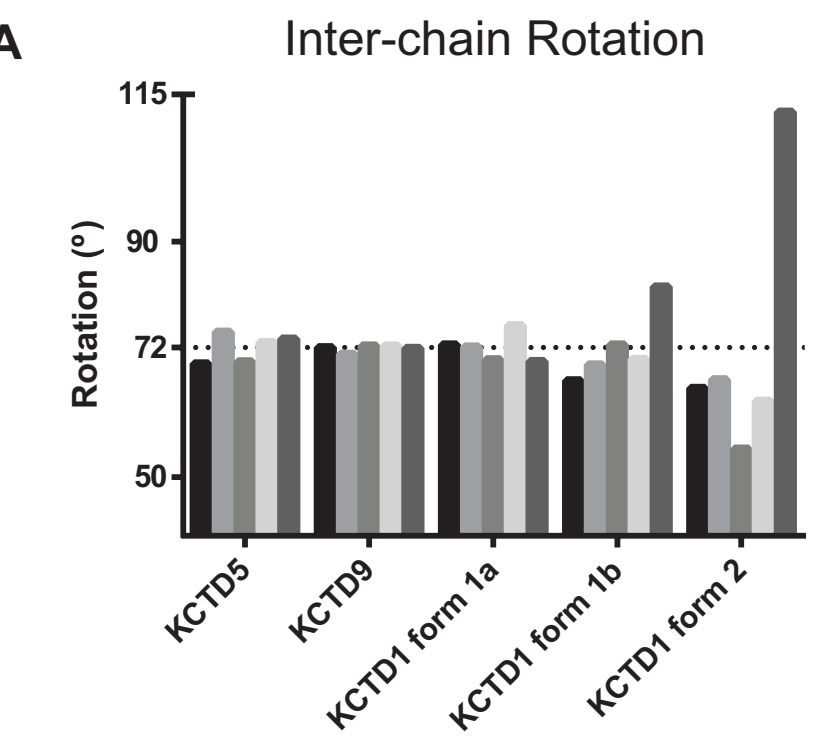

C

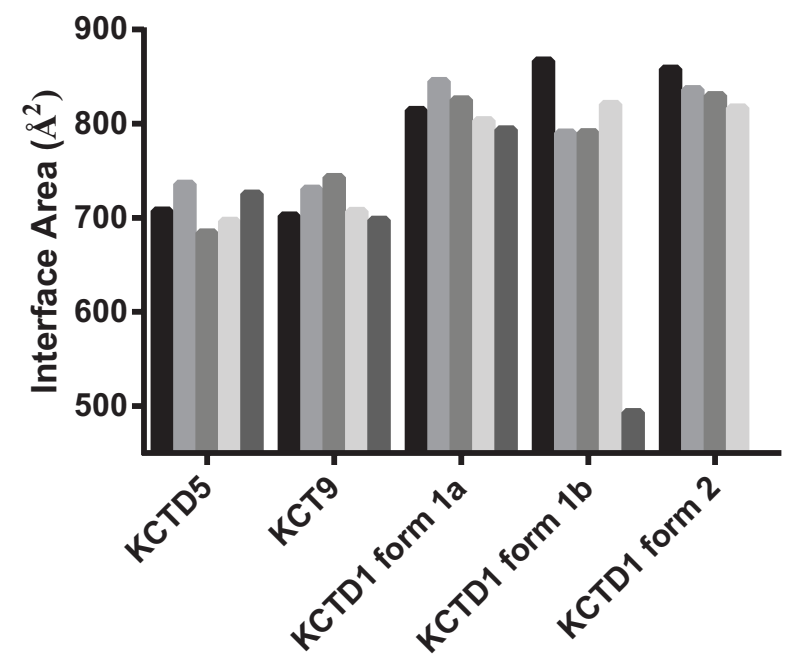

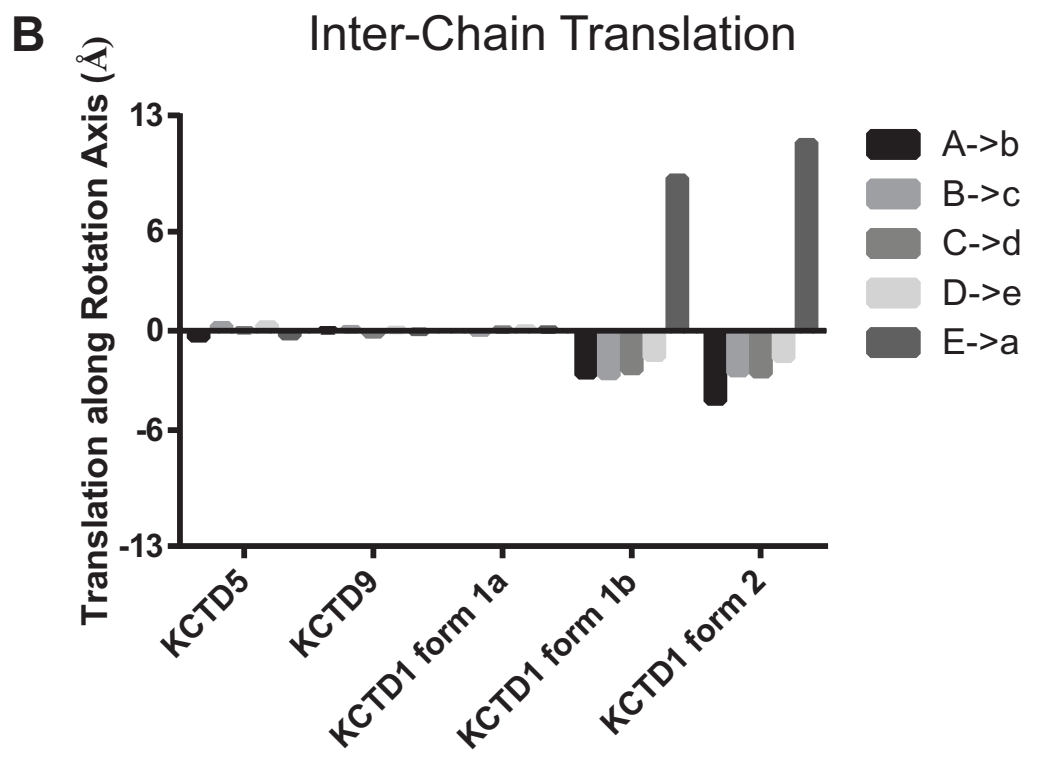

D
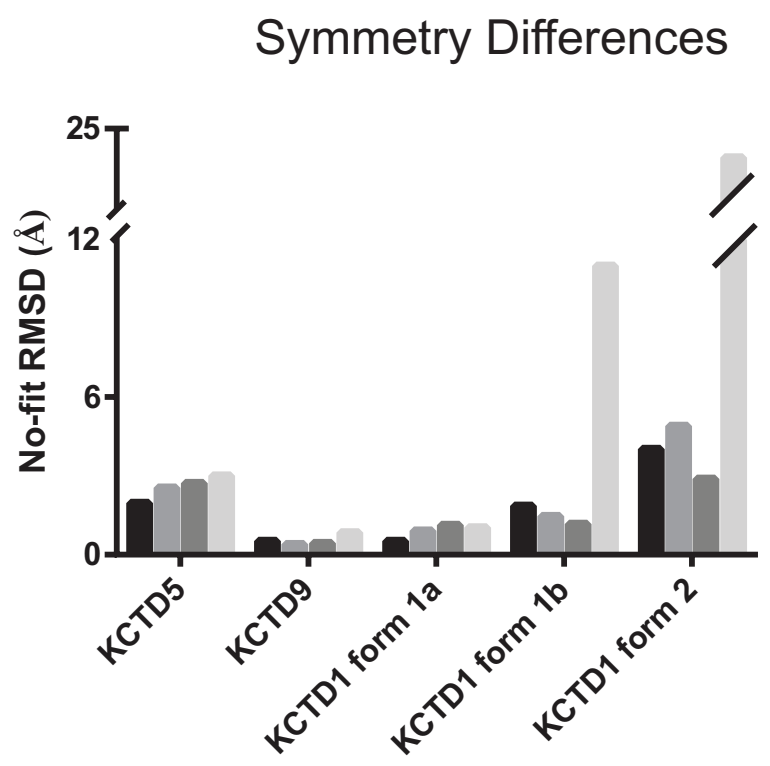

B vs. C

B vs. D

B vs. E

B vs. A 
Figure 3
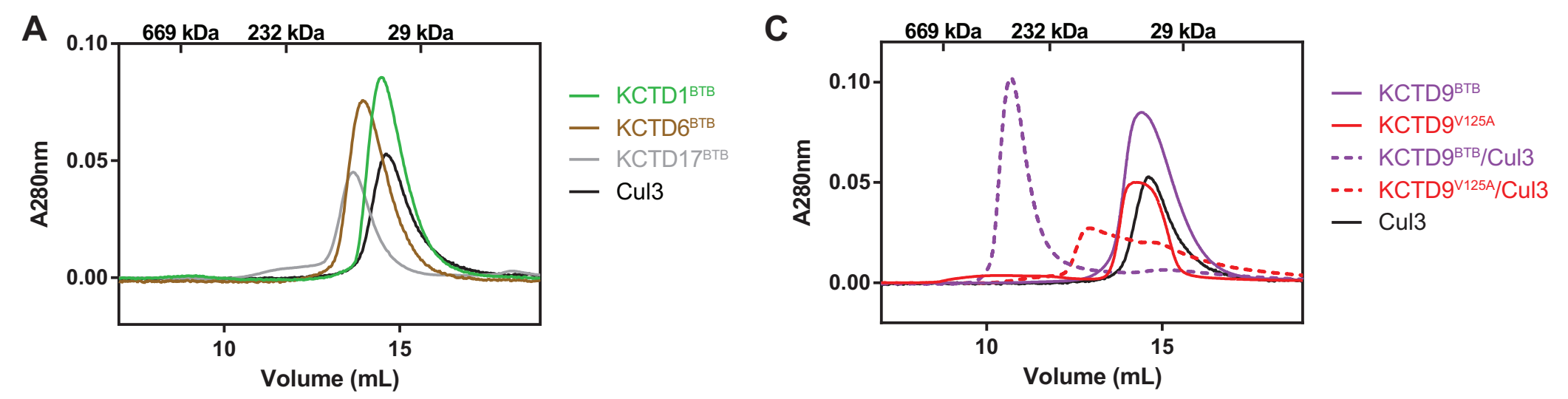

B
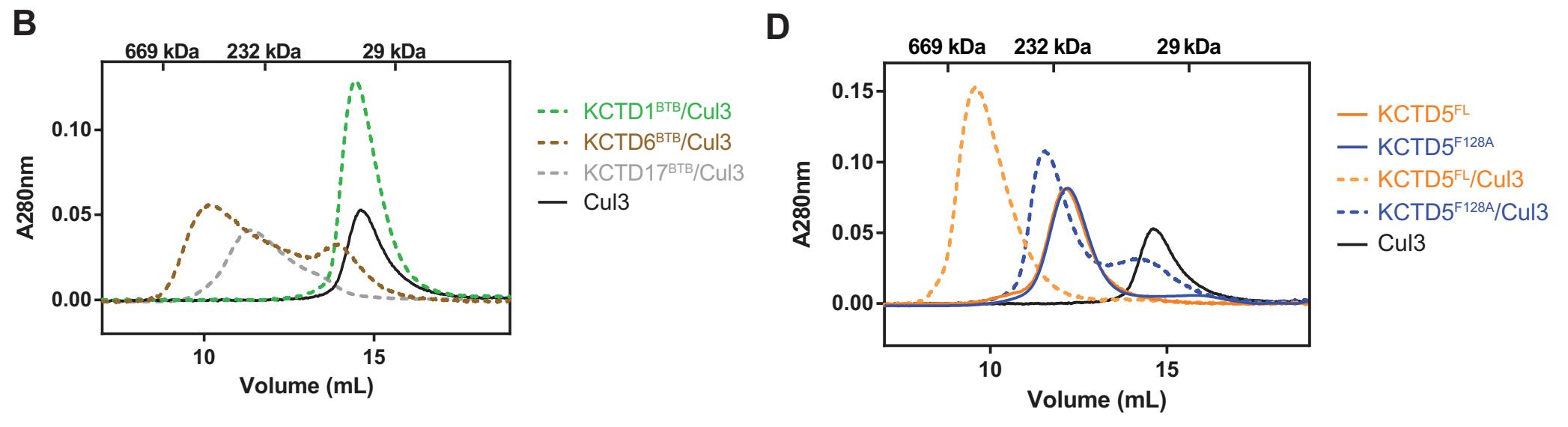

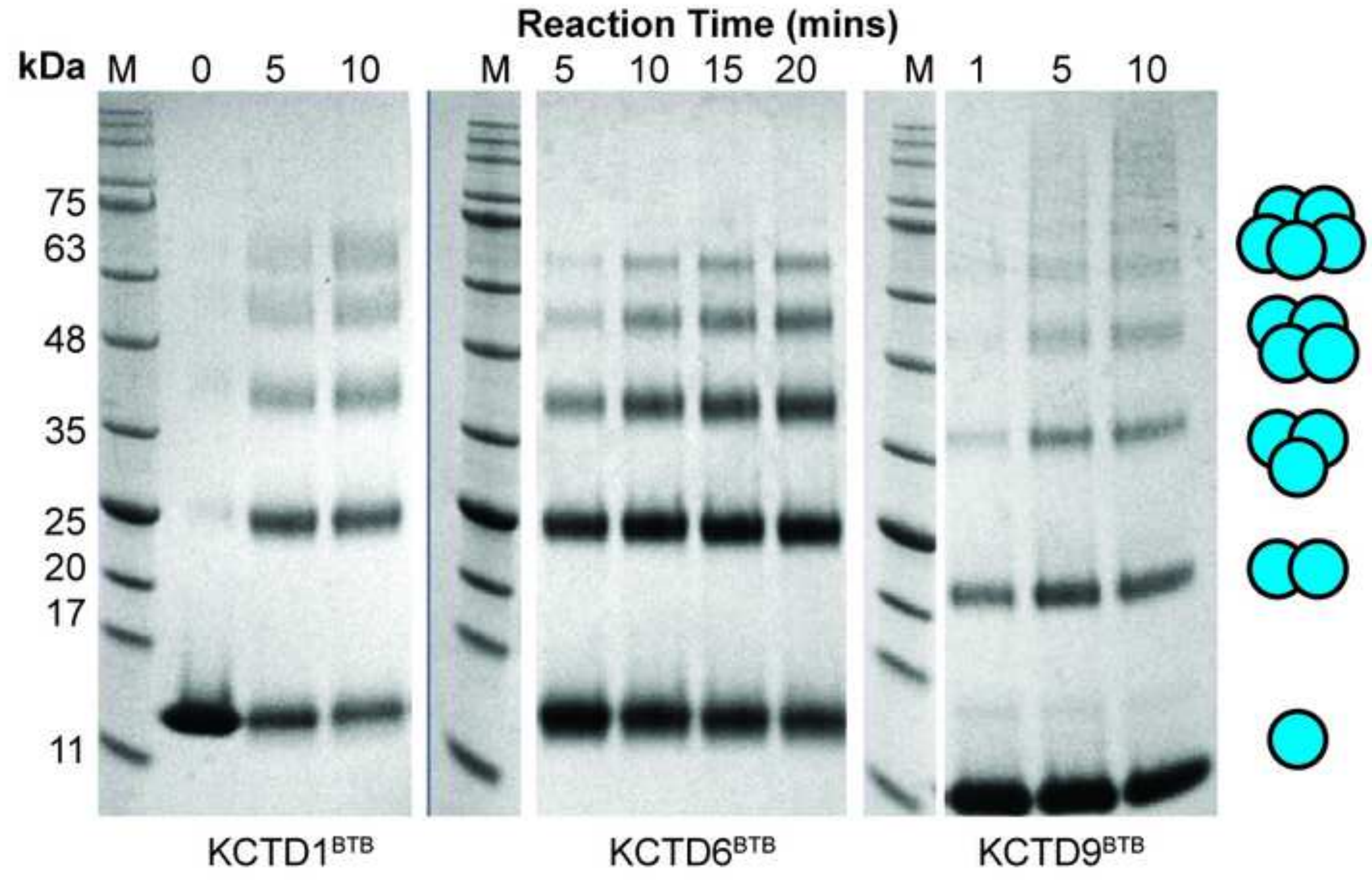

A KCTD1 ${ }^{\mathrm{BTB}}$
B $\mathrm{KCTD}^{\mathrm{BTB}}$
C $\mathrm{KCTD} 5^{\mathrm{FL}}$
D $\mathrm{KCTD}^{\mathrm{F} 128 \mathrm{~A}}$
E KCTD6 $6^{\mathrm{BTB}}$

Time (mins)
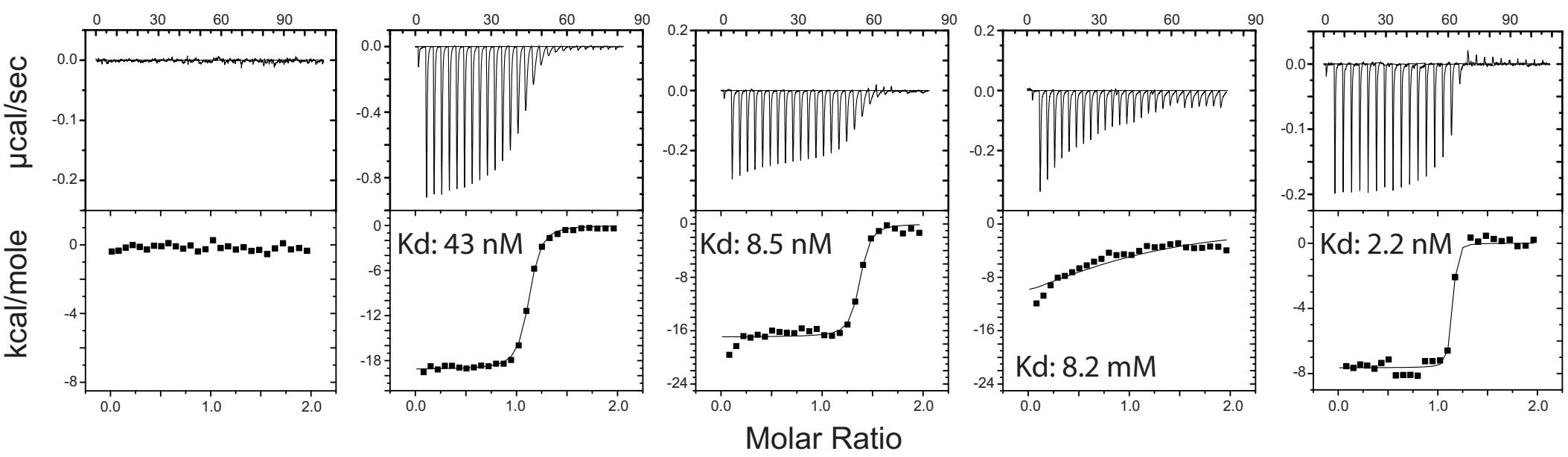

F KCTD9втв

G KCTD9v125A

H KCTD16 ${ }^{\text {BTB }}$

I KCTD17 $7^{\text {BTB }}$

Time (mins)

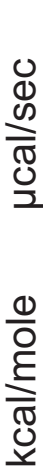
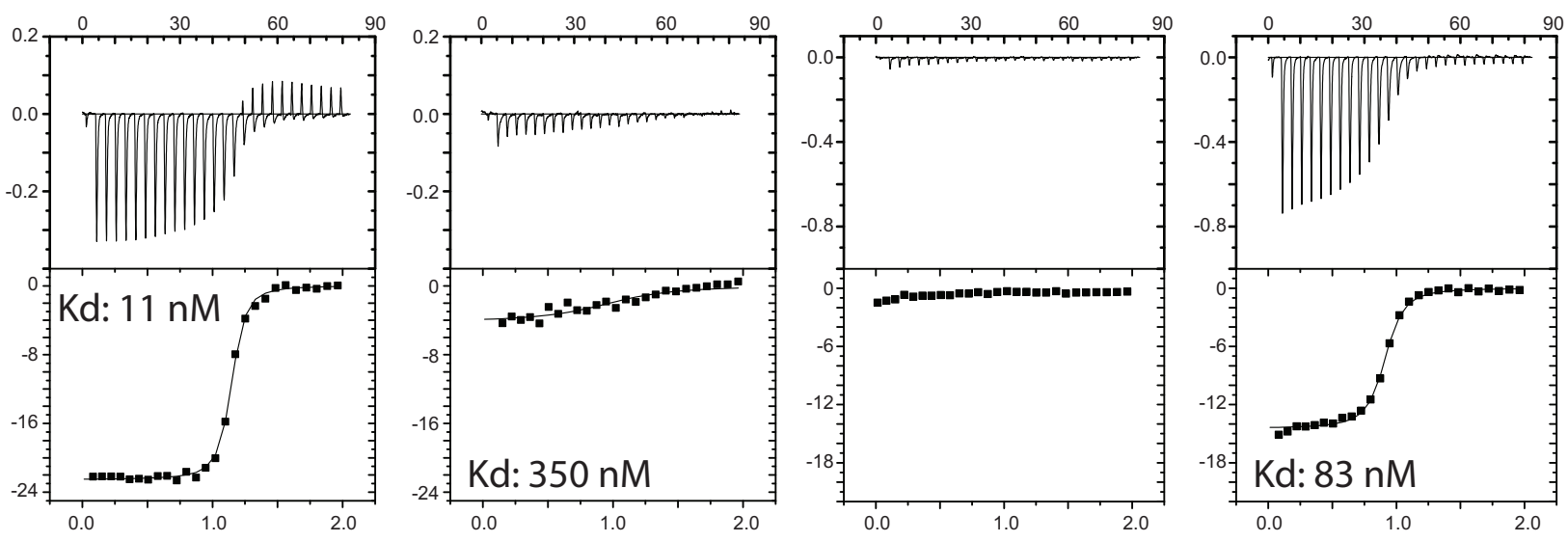

Molar Ratio 
A
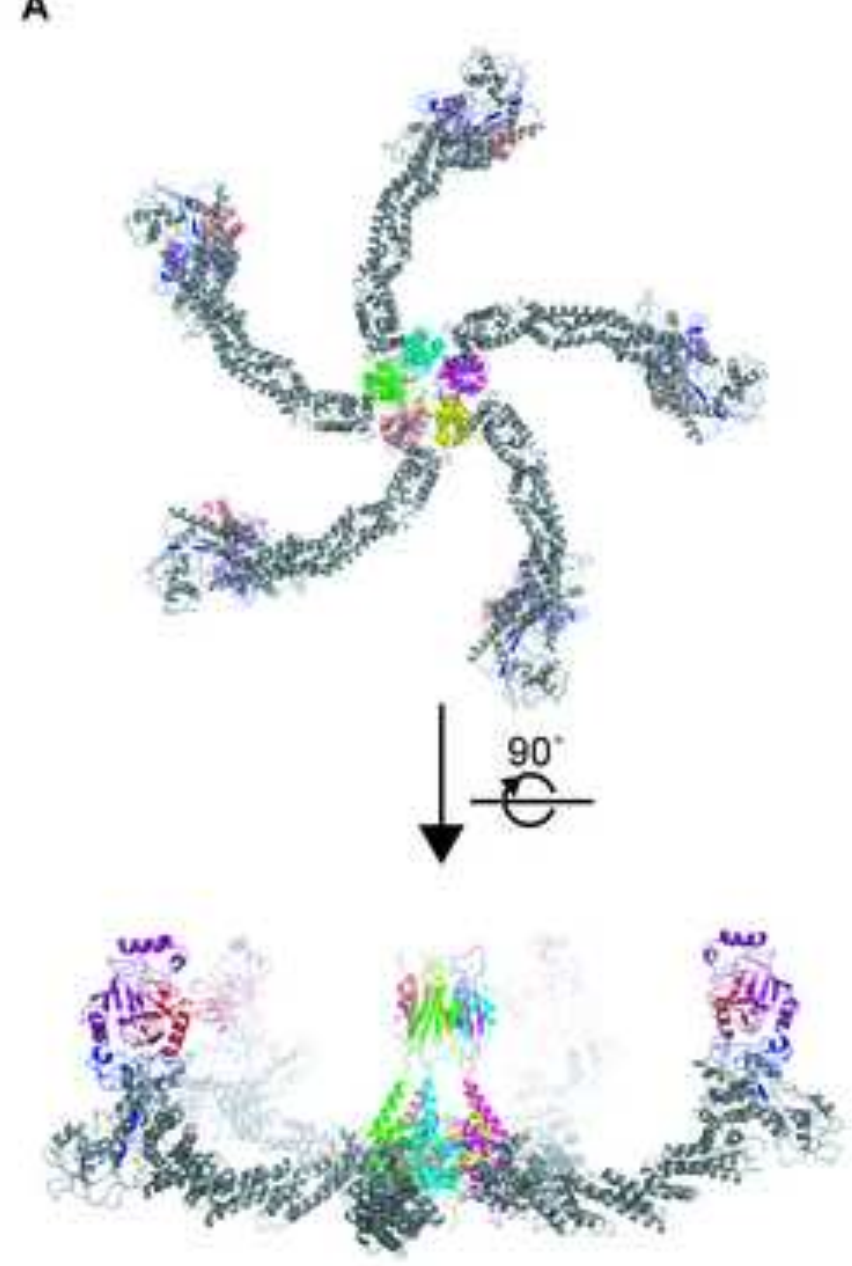

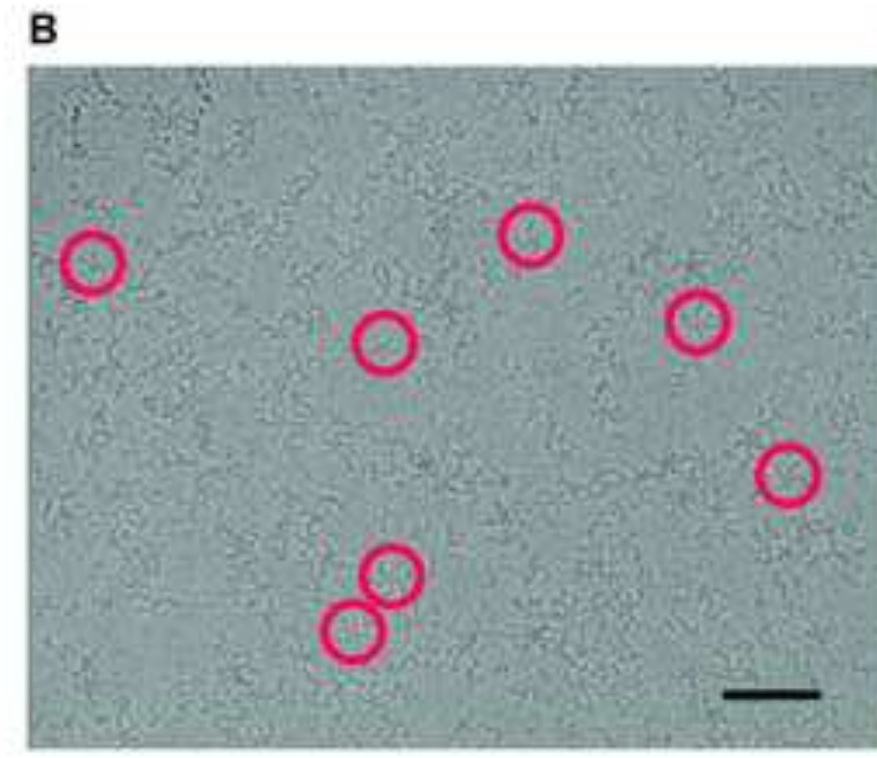

c

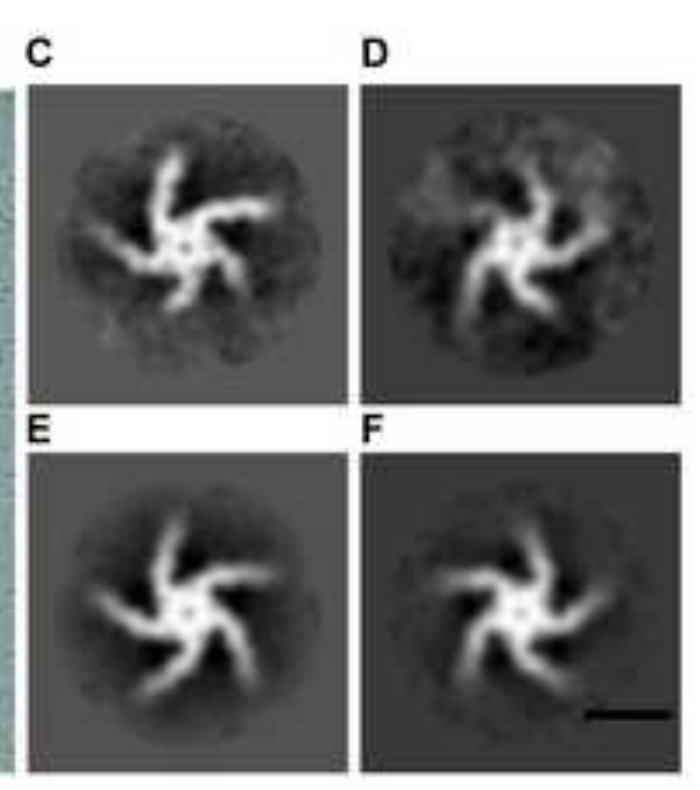

G

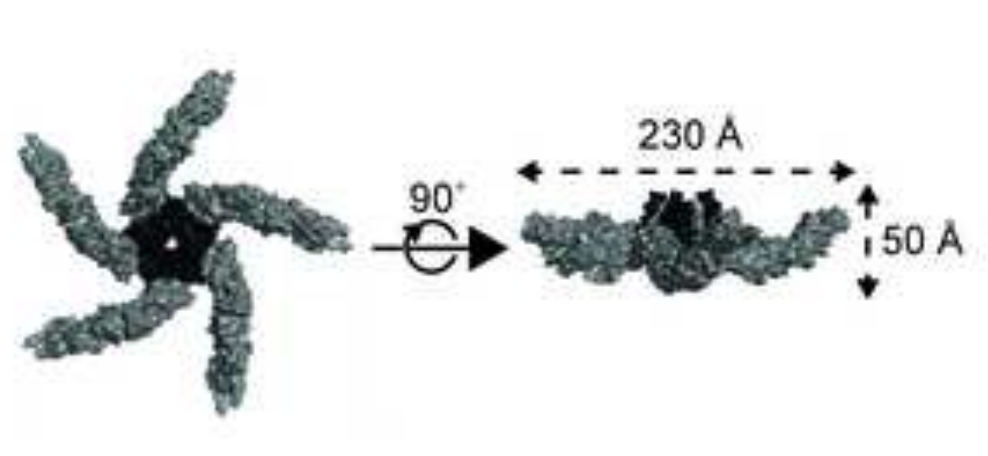

H

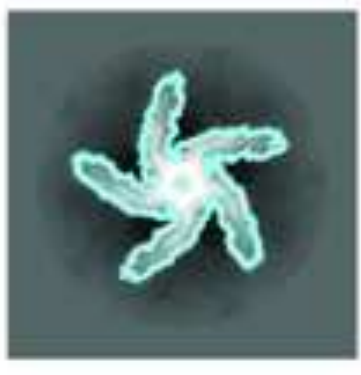

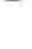

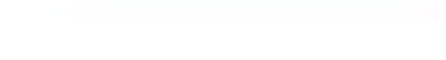


A
KCTD chain E
KCTD chain A
C-term
C-term

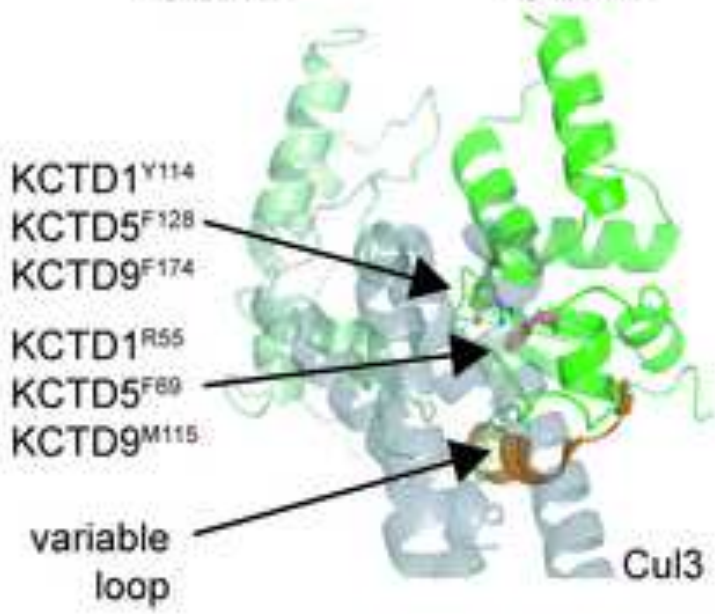

B

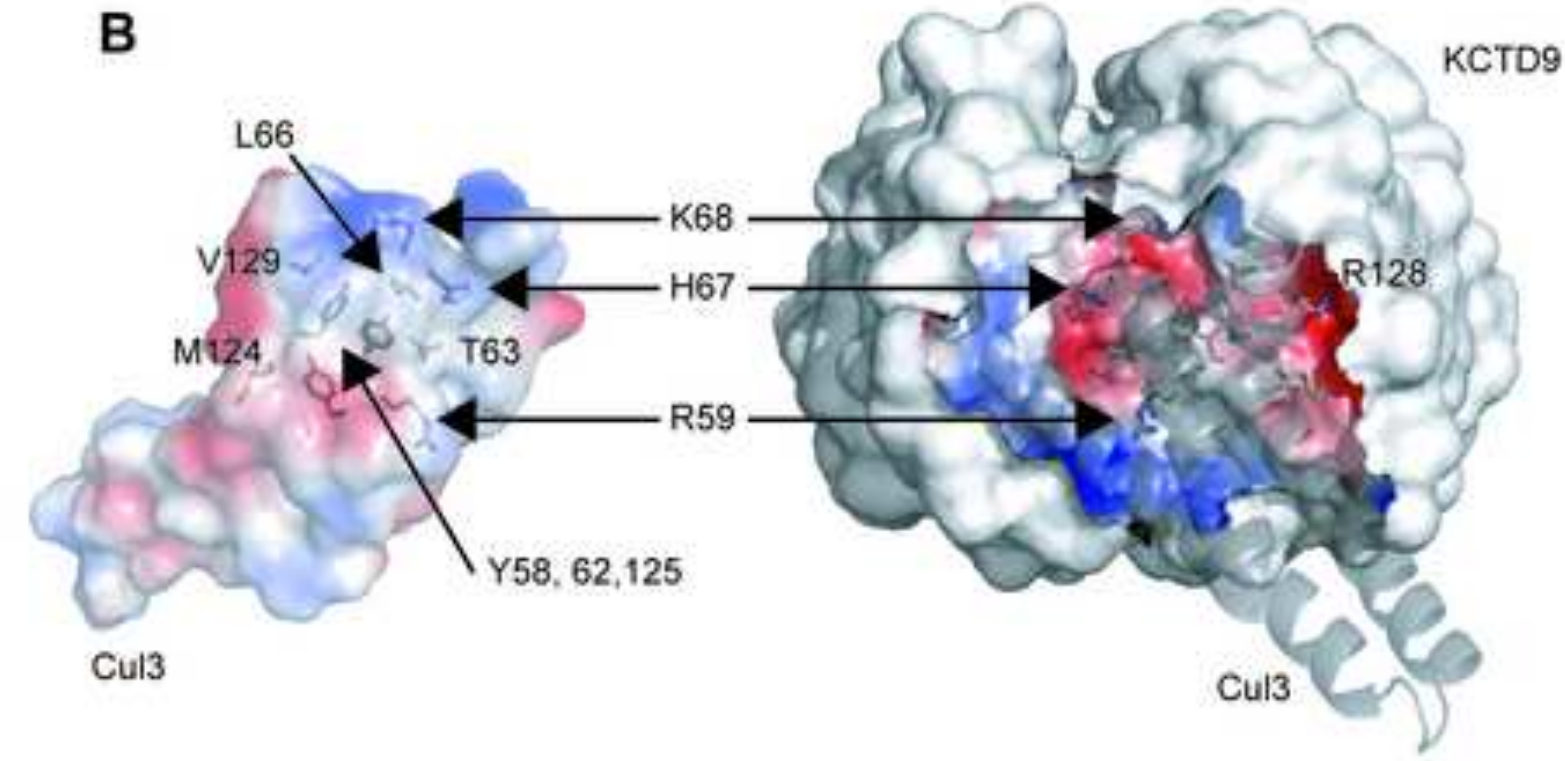

C

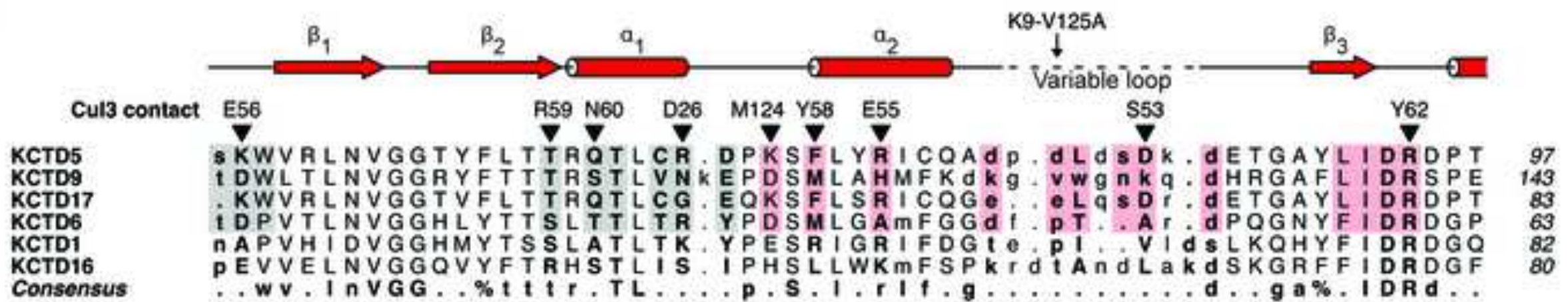
P. wv. InvGG..\%ttir.TL....

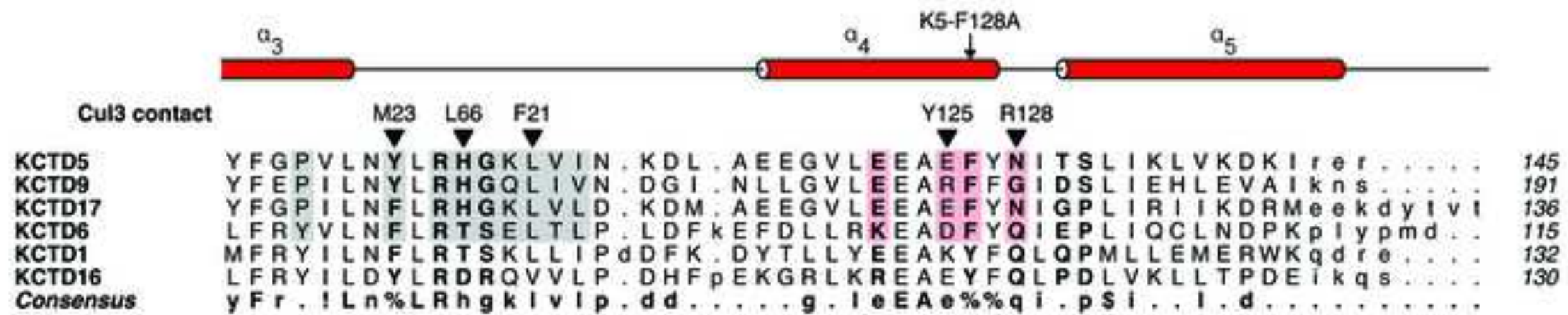


A
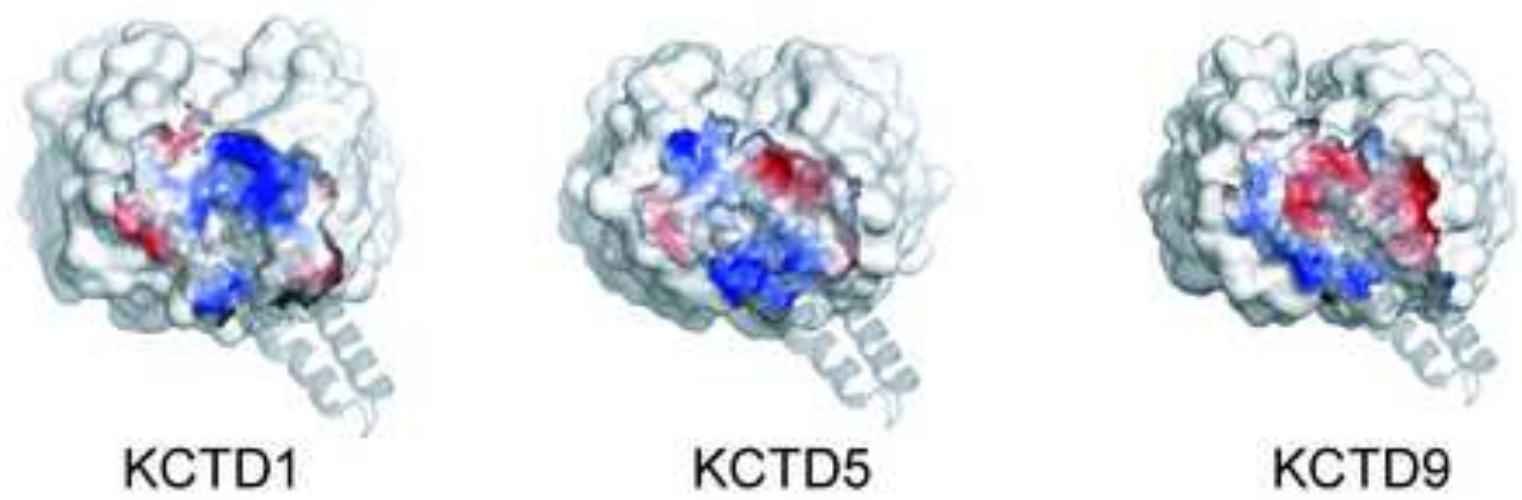

B

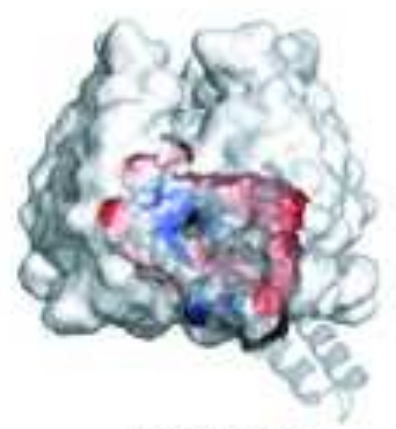

KCTD6

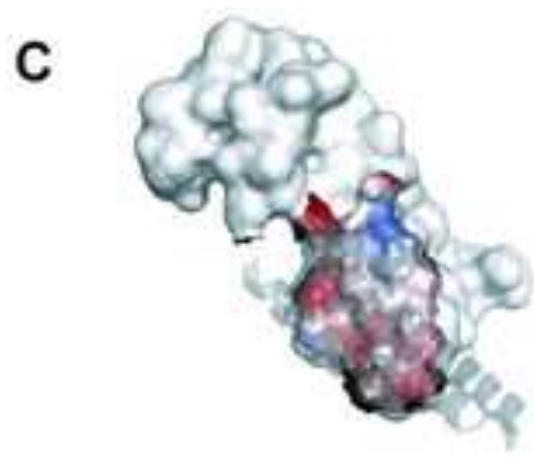

KLHL3

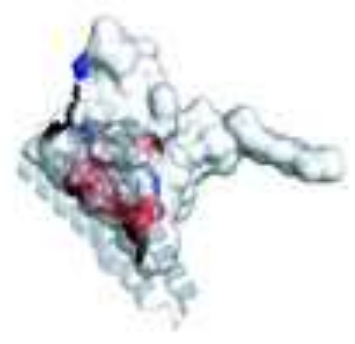

Keap1

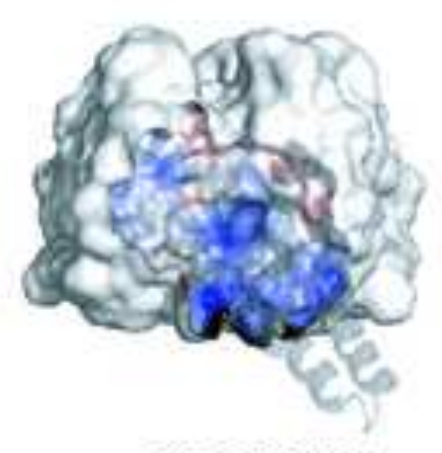

KCTD16

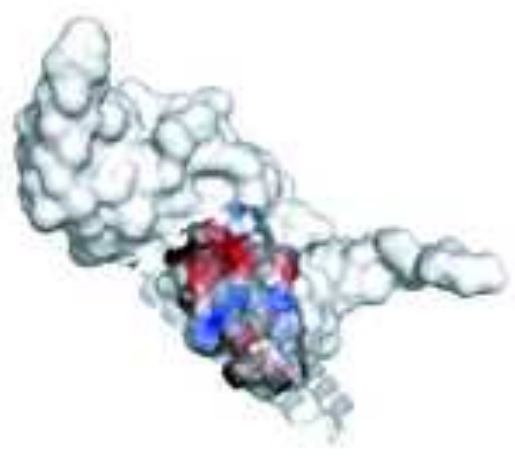

$$
\text { KLHL11 }
$$

D

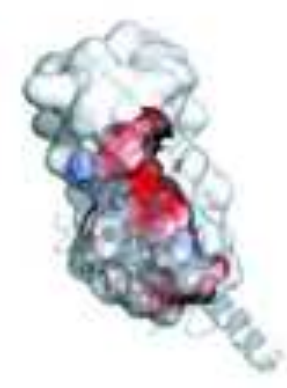

Skp1-2/Cul1
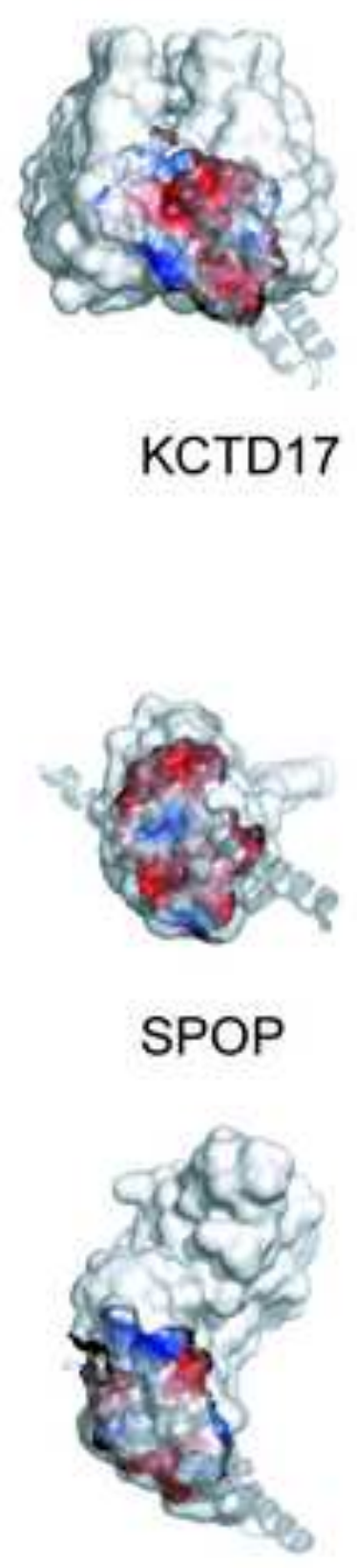

Socs2-EloA/Cul5 
A

4. Variable loop

Phix-E

KCTDS

SPOP

KLHL3

KLHLI1

KEAP1
Consensus
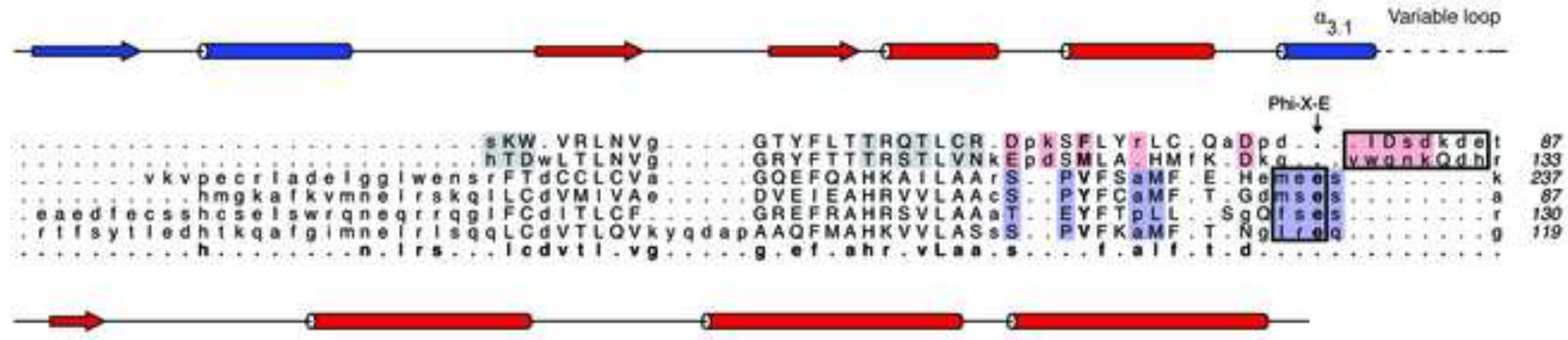

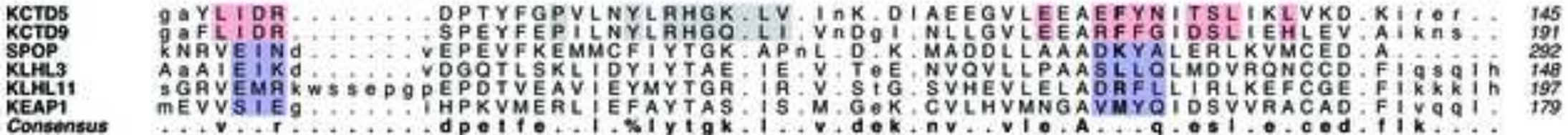

B

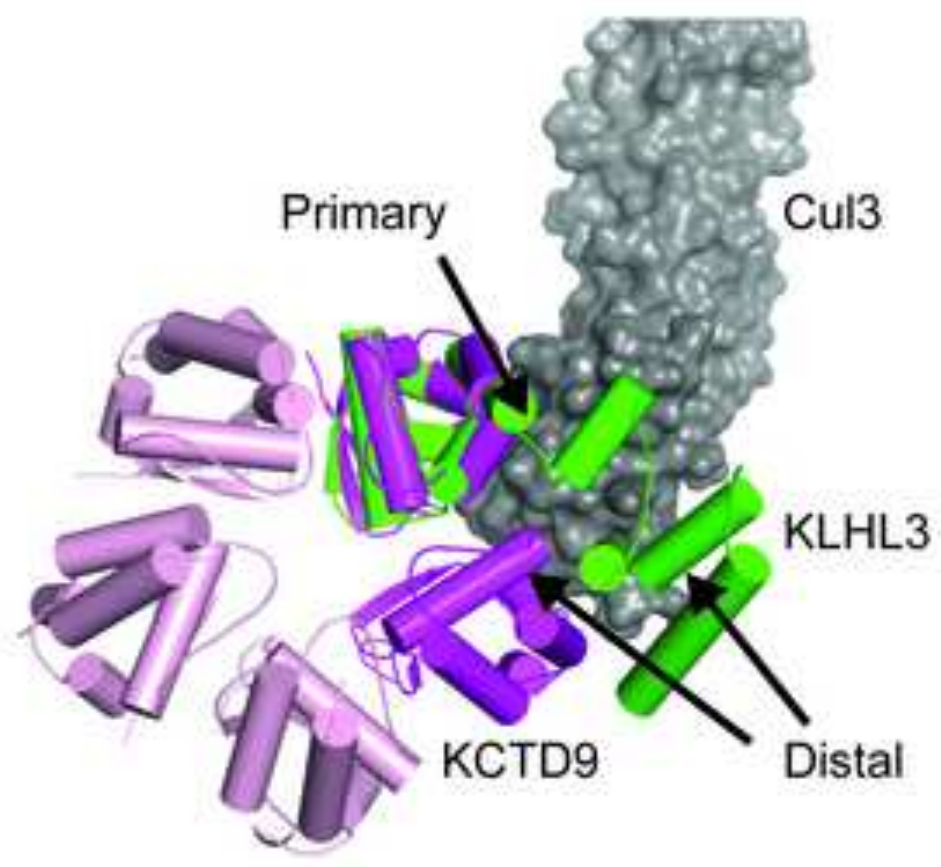




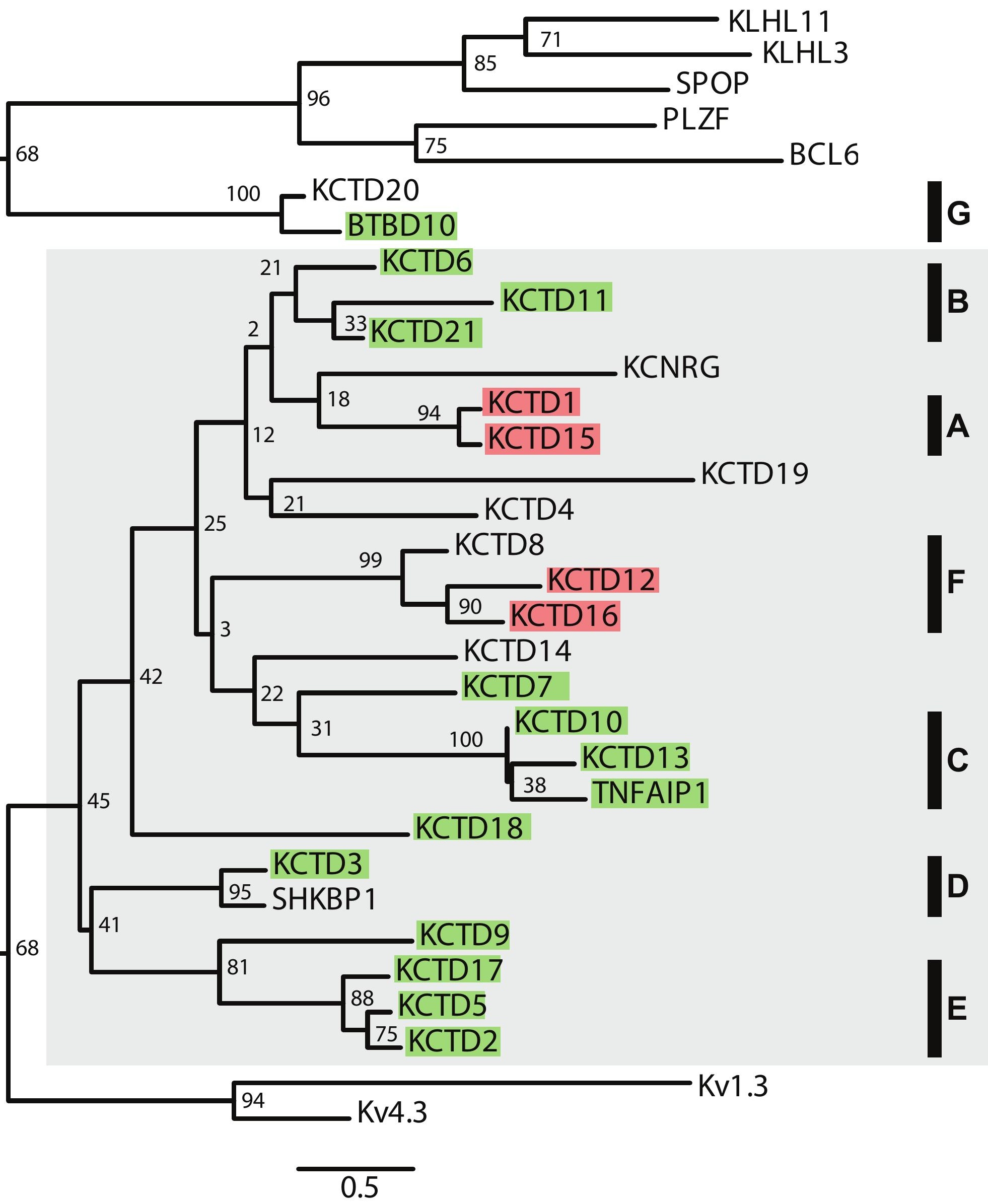

